\title{
The Theory of Teichmüller Spaces - A View Towards Moduli Spaces of Kähler Manifolds
}

Georg Schumacher

\section{Contents}

Introduction

A. Teichmüller Theory

1. Teichmüller's Approach

1.1 Quasiconformal Mappings

1.2 Teichmüller Deformations

1.3 The Moduli Space.

2. The Analytic Structure of the Teichmüller Space

2.1 Real Analytic Theory

2.2 The Tangent Space of $\mathcal{T}_{g}$ and its Complex Structure

3. Hyperbolicity of the Teichmüller Space

4. The Petersson-Weil Metric

4.1 Basic Properties

4.2 The Petersson-Weil Metric for Families of Singular Riemann Surfaces

5. The Curvature of the Petersson-Weil Metric ....................

5.1 Ahlfors' Results .

5.2 Bounds of the Curvature.

5.3 The Curvature of the Petersson-Weil Metric for Singular Families

6. Harmonic Maps and Teichmüller space

7. The Compactified Moduli Space

7.1 Properties of $\overline{\mathcal{M}}_{p}$

7.2 The Petersson-Weil Class on $\overline{\mathcal{M}}_{p}$ as Chern Class of a Positive Line Bundle

B. Moduli Spaces of Compact Kähler Manifolds

8. Existence Theorems for Moduli Spaces of Polarized Kähler Manifolds

9. Moduli Spaces of Kähler-Einstein Manifolds

9.1 The Generalized Petersson-Weil Metric

9.2 A Positive Line Bundle on the Moduli Space

10. Moduli Spaces of Extremal Kähler Manifolds

10.1 Construction of the Moduli Space

10.2 The Generalized Petersson-Weil Metric

10.3 Positive Line Bundles 


\section{Introduction}

Over the last five decades, beautiful results have been proved in the subject of Teichmüller theory. Recently this area has been influenced by the spirit of analytic and algebraic geometry as well as complex differential geometry. Deformation theory of compact complex manifolds was created in a seemingly independent way. Its methods are significantly different and, as opposed to its classical counterpart, deformation theory only provides a local solution of the classification problem. A (coarse) moduli space, i.e. a global parameter space for complex structures exists only under certain assumptions. The aim of this article is to discuss some aspects of Teichmüller theory and their relationships to recent results on moduli of compact complex manifolds.

In his paper of 1857 "Theorie der Abel'schen Functionen" Riemann counted the number of parameters of isomorphism classes of algebraic equations in two variables i.e. classes of compact Riemann surfaces up to biholomorphic equivalence.

Some eighty years later Teichmüller realized that these parameters are real coordinates on a cell. Its points are quadratic holomorphic differentials on a surface which has been choosen: chosen as base point. The distinguished surface is related to the other points of the Teichmüller space by quasiconformal mappings. This relationship yielded a natural metric on the parameter space of Teichmüller. His last paper in this area was devoted to the complex structure of this space. The foundation of modern Teichmüller theory is due to Ahlfors and Bers. In particular they endowed the Teichmüller space with a complex structure and constructed a universal holomorphic family of Riemann surfaces.

The Teichmüller metric can be understood from the complex point of view from Royden's result which shows that it is the hyperbolic metric. Royden also uses this result to prove that the group of biholomorphic mappings of the Teichmüller space is just the Teichmüller modular group.

André Weil examined the Teichmüller family from the point of view of deformation theory. He proposed a certain metric on the moduli space and conjectured it to be Kähler. Petersson had already considered the corresponding inner product in the context of automorphic forms. In the hindsight this Petersson-Weil metric seems to have been the turning point, namely for the introduction of methods of complex differential geometry into the study of moduli space of compact Riemann surfaces. After proving the Kähler property, Ahlfors showed in 1961 that the holomorphic sectional and Ricci curvatures were negative. In 1974 Royden conjectured a precise upper bound for the holomorphic sectional curvatures of the Petersson-Weil metric. This, along with the negativity of the sectional curvature was established independently by Wolpert and Tromba in 1986. Its strong negativity in the sense of Siu was proved in [SCH 1986].

Another characterization of the Teichmüller space is the space of metrics of constant curvature -1 modulo diffeomorphisms. This led to an approach based on harmonic maps. It was initiated by Gerstenhaber and Rauch with 
more recent developments due to Fischer, Tromba, M. Wolf, and Jost. One of the main results is that the energy functional on a space of harmonic maps yields a potential for the Petersson-Weil metric.

If one is willing to disregard questions involving universal families, then one may consider the space of isomorphism classes of compact Riemann surfaces, i.e. the quotient of the Teichmüller space by the Teichmüller modular group. This is referred to as the moduli space. It carries an additional structure of a quasi-projective variety.

The compactification of the moduli space can be looked upon from different viewpoints: one is the algebraic viewpoint due to Mumford, another analytic approach is by Kleinian groups. Both involve deformations of singular and punctured Riemann surfaces. It turns out that the completion of the moduli space with respect to the Petersson-Weil metric is its (usual) compactification. This followed from estimates proven by H. Mazur. Estimates for the curvature tensor near the compactifying divisor are contained in [SCH 1986].

In terms of Fenchel-Nielsen coordinates, the Petersson-Weil form has the surprisingly simple shape of the standard symplectic form - a result shown by Wolpert in 1985. Comparing the different differentiable structures on the compactified moduli space which are induced by the usual holomorphic and the Fenchel-Nielsen coordinates, Wolpert proved that the Petersson-Weil class can be extended to the compactification. In fact, the Petersson-Weil form possesses local continuous potentials near the boundary. An essential result in this regard is a fiber-integral formula for the Petersson-Weil form. His result that the Petersson-Weil class on the compactified moduli space is (up to a numerical constant) the Chern class of an ample line bundle unifies the approach from algebraic geometry and Kähler geometry. This ample line bundle is closely related to the bundle that arises as the determinant of a certain direct image sheaf (the Knudsen-Mumford approach).

Based upon the powerful methods of deformation theory one can construct even in the case of non-algebraic Kähler manifolds a moduli space. For this, the notion of a polarization of a projective variety, which is closely related to its realization as a submanifold of some projective space, had to be replaced by the assignment of a Kähler class. In higher dimensions there occur additional complications. In the first place there no longer exists a global family. Even if the base space of a holomorphic family is treated as an analytic space-germ, there is in general no natural action of the automorphism group of the distinguished fiber on the base. This is contrary to the classical situation, where the Teichmüller modular group (more precisely the isotropy group of a point) comes from the automorphism group of the corresponding Riemann surface. Here the Teichmüller family yields a local universal deformation, and locally the moduli space is the quotient of this group. A general compact complex manifold only possesses a "versal" rather than universal deformation. This fact is related to the occurence :occurrence of locally analytic subsets of the base, where all fibers are isomorphic. An identification of such points always yields a non-hausdorff topology. In fact there exist very simple examples of this kind. 
Even if the manifolds in question possess universal deformations, this is not sufficient for the existence of a moduli space.

A construction of the moduli space of polarized Kähler manifolds of a certain type or with additional structure can often be based on a simple criterion: Assuming the existence of versal deformations which is an obvious necessary prerequesit: prerequesite a coarse moduli space exists, if roughly speaking given any two families of complex manifolds and given any two sequences of pairwise isomorphic fibers there are subsequences of isomorphisms which converge to an isomorphism of the limit fibers [SCH 1983/1984]. The assumptions of the criterion were first verified in a situation which is closely related to the classical one, where biholomorphic mappings of compact Riemann surfaces are exactly the (orientation-preserving) isometries with respect to metrics of constant curvature. If in general the polarization can be realized by a distinguished Kähler metric with the above relationship between isomorphisms in the analytic category and isometries, the assumptions are automatically satisfied. In this way a moduli space for polarized Kähler manifolds with vanishing first real Chern class was constructed [SCH 1983]. This uses the unique Ricci-flat metrics according to Yau's solution of the Calabi problem. More generally, later the moduli space of polarized non-uniruled Kähler manifolds was constructed by Fujiki in [FU 1984] and in [SCH 1984].

For Riemann surfaces the Petersson inner product for quadratic holomorphic differentials yields an inner product on the cotangent space of the Teichmüller space. In the general setting by means of the Kodaira-Spencer map, this product on the tangent space is just the $L^{2}$-inner product of harmonic representatives of first cohomology classes with values in the tangent sheaf. According to Koiso, one may treat canonically polarized manifolds and compact polarized Kähler manifolds with vanishing first real Chern class in an analogous way by using Kähler-Einstein metrics. The variation of such metrics in a family gives in fact rise to a Kähler metric on the smooth part of the base.

Work on moduli spaces in higher dimensions uncovered a number of natural questions. First, could the generalized Petersson-Weil form be represented as a fiber integral? This would yield the Kähler property even at singular points, and also the existence of a Kähler potential (a consequence of a theorem of Varouchas). Second, can one find a hermitian line bundle on the moduli space of canonically polarized manifolds say, with curvature equal to the Petersson-Weil metric? Third, is there a natural Kähler metric on the moduli space of compact polarized Kähler manifolds? These questions were treated in [F-S 1988], where for example a fiber integral formula was proved. To obtain the hermitian line bundle on the moduli space of canonically polarized manifolds the direct images of powers of the relative canonical bundles had to be replaced by determinant bundles of certain virtual bundles related to the canonical one equipped with the Quillen metric. Its curvature is the Petersson-Weil form. The link with the fiber-integral formula for the generalized Petersson-Weil form was furnished by the generalized Riemann-Roch formula (for Chern forms rather than classes) by Bismut, Gilet and Soulé which also holds for singular base spaces. 
The third question is treated in the following manner: Equip a compact polarized Kähler manifold with an extremal Kähler form (which represents the polarization). The general framework for such considerations is given by the above existence theorem for a moduli space of compact polarized Kähler manifolds which are not uni-ruled. The latter condition guarantees that in particular the components of the automorphism groups are compact. On such manifolds, extremal metrics are just those of constant scalar curvature. The general criterion for the existence of a moduli space applies.

However, in the case of moduli spaces of extremal Kähler manifolds the $L^{2}$-inner product of harmonic representatives of Kodaira-Spencer classes does not give rise to a Kähler metric on the moduli space (at least this is very unlikely). What one needs is a close relationship between the variation of the complex structures in a family of compact Kähler manifolds on one hand (i.e. a representative of the Kodaira-Spencer class), and on the other hand the variation of the distinguished Kähler metrics on these fibers which is described by a certain symmetric tensor). The key for the solution lies in the Berger-Ebin formula for the variation of the metric tensor in a family of Riemannian metrics with constant scalar curvature and Calabi's investigations. In the Kähler case all terms except for: except one of this expression vanish. This yields a forth order elliptic equation. This equation generalizes and replaces harmonicity.

The generalized Petersson-Weil metric is now defined through the $L^{2}$-inner product of such "harmonic" representatives of Kodaira-Spencer classes. The Kähler property can be directly verified, and just as in the Kähler-Einstein case a fiber-integral formula yields the Kähler property even at singular points of the moduli space. One should mention that computations are done with respect to the base of a universal deformation: the moduli space is locally the quotient of such by a finite group of automorphisms. The Petersson-Weil form descends to the quotient, but its potential may only be continuous. For integer-valued polarizations the moduli space is equipped with a hermitian line bundle whose curvature is the Petersson-Weil form. This is achieved in the following way: (1) classify extremal manifolds with isomorphism classes of ample line bundles, (2) construct a hermitian bundle and a Petersson-Weil form on the respective moduli space, (3) descend to the desired moduli space by fiber integration and determinant bundle.

A consequence is that all (connected) compact complex subspaces of the moduli space of rationally polarized, extremal Kähler manifolds (including the cases of canonically polarized manifolds and of Kähler-Einstein manifolds with positive curvature) are projective. 
Chapter A

\section{Teichmüller Theory}

\section{Teichmüller's Approach}

Oswald Teichmüller's most influential article "Extremale quasikonforme Abbildungen" [TE 1939] contains essentially what nowadays is called "Teichmüller-theory" - the classification of Riemann surfaces by means of a parameter space.

He chose a somehow indirect approach: The classification of Riemann surfaces up to biholomorphic maps leads to the so-called "(coarse) moduli space" which turned out to contain singularities. He introduced a refined equivalence relation instead. First a Riemann surface $X_{0}$ had to be fixed. Variable surfaces $X$ had to be equipped with an orientation-preserving homeomorphism $f$ from $X_{0}$ to $X_{1}$. Two such "marked" surfaces are identified, if a biholomorphic map from $X_{1}$ to $X_{2}$ takes such a homeomorphism $f_{1}$ to a homoeomorphism: homeomorphism homotopic to $f_{2}$.

He was able to show that the set of such equivalence classes carries the differentiable structure of a cell contained in a euklidean: euclidean space, whose dimension had already been computed by Riemann in 1857 . From the beginning of his construction this set was equipped with an intrinsic metric which he also related to a Finsler metric. Both the space and the metric bear his name. The Teichmüller space of compact Riemann surfaces of genus $p$ is denoted by $\mathcal{T}_{p}$.

At the end the moduli space of Riemann surfaces was determined as the quotient of the Teichmüller space by a discrete group of automorphisms, the Teichmüller modular group.

Teichmüller reduced the classification problem to constructing a distinguished homeomorphism from the reference surface to a given one - this homeomorphism was to be the so-called extremal quasiconformal map. Considering a complex structure on a Riemann surface as arising from a Riemannian metric with an arbitrary scalar factor, he introduced quadratic differentials as links between extremal quasiconformal maps and conformal structures.

In "Veränderliche Riemannsche Flächen" [TE 1944] Teichmüller developed ideas to equip the Teichmüller-space with a complex structure and a holomorphic family of Riemann surfaces.

One should mention that he also regarded at the same time non-orientable surfaces for which certain results also hold. 


\subsection{Quasiconformal Mappings}

Until Teichmüller realized the power of the notion of quasiconformal mappings, these had been more of a side-issue of geometric function theory, a generalization of holomorphic functions. At some place in [TE 1939] he shortly reviews the known aspects. He remarks that quasiconformal mappings should be used to study holomorphic mappings between Riemann surfaces $\mathcal{A}$ and $\mathcal{B}$, but not like approximating these by quasiconformal ones. One should rather consider quasiconformal maps from a fixed surface $\mathcal{C}$ to these.

Notion of Quasiconformal Mappings and the Problem of Grötzsch. Quasiconformal mappings had been introduced by Grötzsch in 1928. He showed a Picard type theorem but also solved an elementary problem about extremal mappings which ultimately lead to the Teichmüller theorems.

For any differentiable map $f: G \rightarrow G^{\prime}, w=f(z)$ its dilatation at a point $z$ is defined as

$$
K_{f}(z)=\frac{\left|f_{z}(z)\right|+\left|f_{\bar{z}}(z)\right|}{\left|f_{z}(z)\right|-\left|f_{\bar{z}}(z)\right|}
$$

and plainly its dilatation

$$
K[f]=\sup K_{f}(z) .
$$

Grötzsch showed that for any diffeomorphism $f$ of rectangles in the complex plane, taking vertices to vertices in a fixed order, the dilatation $K[f]$ is bounded from below by a positive constant $K_{0}$ which is only attained for affine maps which are the extremal quasiconformal maps for this problem.

The idea of his proof is now mostly called "length-area" argument. He considers the image of a horizontal line from one edge to the other. Its length, as a function of the vertical coordinate, is integrated. This integral satisfies an obvious inequality in terms of the lenghts: lengths of the edges. On the other hand this area integral can be estimated from above by an integral involving the jacobian and the dilatation.

Differentiable orientation preserving homeomorphisms $w=f(z)$ of domains in the complex plane or between Riemann surfaces with bounded dilatation are called quasiconformal, and, more generally, if absolutely continous: continuous on lines and differentiable almost everywhere (with bounded dilatation).

Teichmüller allowed bordered Riemann surfaces with boundary curves and isolated boundary points ("Hauptbereiche") — we will restrict ourselves later to the case, where no boundary curves, just punctures, are present. The nonoriented case will be disregarded here.

Examples of Extremal Quasiconformal Mappings. Before his article [TE 1939] appeared, extremal quasiconformal mappings had been determined in some particular cases. Teichmüller considered the analogue of the problem of Grötzsch for annuli in the complex plane and arrived at a similar result: The lower bound 
for the dilatation of a map between annuli is the quotient of the respective moduli and attained for an affine map. (Here the modulus of $\{z \in \mathbb{C} ; r<|z|<R\}$ is by definition $M=\log (R / r)$, which is the conformal invariant of the annulus).

As for compact Riemann surfaces, he looked at quasiconformal mappings of compact tori. The classifying space of marked compex: complex tori was known to be the upper half plane $H$ : Any quasiconformal map from a torus with modulus $\omega \in H$ to a torus with period $\omega^{\prime} \in H$ has a dilatation bounded from below by $\Im\left(\omega^{\prime}\right) / \Im(\omega)$. Provided the real parts of $\omega$ and $\omega^{\prime}$ are equal, this number is the dilatation of an affine map. (In general the lowest dilatation is different but the extremal maps are in any case affine.)

As an example of a punctured Riemann surface he considered surfaces $X$ of genus zero with four points removed. Because of the marking these points are ordered and can be chosen as $0,1, \infty$ and $\lambda$. Any such $X$ is related to a two-sheeted covering of $\mathbb{P}^{1}$ by an elliptic curve with these points as branch points. One can directly establish a biholomorphic mapping from $\mathbb{C} \backslash\{0,1\}$ to the upper half plane sending $\lambda$ to an appropriately chosen modulus of the elliptic curve. In terms of the elliptic curves extremal quasiconformal mappings are induced by affine mappings of the complex plane.

In all of these examples the dilatation of an extremal quasiconformal mapping in the sense of (1.1) is constant.

The Teichmüller Metric. The analysis of the above (and more) examples seems to be the cause for Teichmüller to define a metric on the Teichmüller space. He poses the following question: Given a fixed Riemann surface and an upper bound $C$ for the dilatation which are the quasiconformal maps, i.e. marked Riemann surface to lie within that bound?

Remarkable is the discussion of the torus case. If the period of the reference surface is $\omega$, the estimate $(1 / C) \Im(\omega) \leq \Im\left(\omega^{\prime}\right) \leq C \Im(\omega)$ yields that the range for $\omega^{\prime}$ is an intersection of domains, whose boundary consists of two circular arcs. It is computed to be the non-euklidean: non-euclidean disc with center $\omega$ and radius $\log C$.

The Teichmüller distance of two marked Riemann surfaces is in fact defined in terms of the logarithm of the dilatation.

$$
\tau(P, Q)=(1 / 2) \log \inf \{K[f] ; f: P \rightarrow Q \text { quasiconformal }\}
$$

From this formula and the above discussion it follows immediately that the Teichmüller metric on the space of marked elliptic curves is equal to the hyperbolic metric on the upper half plane.

This principle proved even sound in the case of annuli, where the classifying space consists of all positive real numbers. The Teichmüller distance of two moduli $M$ and $N$ is here

$$
\tau(M, N)=|\log M-\log N| .
$$

For $\mathbb{P}^{1}$ with four punctures the Teichmüller space had been related to $\mathbb{C} \backslash\{0,1\}$. Teichmüller showed, using the result about tori that the above expression (1.3) gives the metric of constant curvature -1 on $\mathbb{C} \backslash\{0,1\}$. In all of 
these examples the construction implies that (1.3) actually defines a distance function.

\subsection{Teichmüller Deformations}

Teichmüller was able to put his hands on the desired extremal quasiconformal mappings. In this way he could identify the Teichmüller space with the open unit ball in the space of quadratic holomorphic differentials with respect to a suitable norm.

Conformal Structures Induced by Holomorphic Quadratic Differentials. A quasiconformal map can be visualized locally by the associated horizontal and vertical foliations - the images of the lines $\Re(z)=$ const and $\Im(z)=$ const. Teichmüller observed that any quasiconformal map can be recovered up to a conformal map from these foliations and the dilatation. He first argued heuristically that an extremal quasiconformal map ought to have constant dilatation. On the other hand he explicitly constructed extremal mappings from quadratic differentials and prescribed dilatation $K>1$.

Let $\phi(z) d z^{2}$ be a holomorphic quadratic differential given in local coordinates and $p_{0}$ a point. Then

$$
\zeta=\int_{p_{0}}^{p} \sqrt{\phi d z^{2}}
$$

defines a local coordinate about $p_{0}$ if $\phi\left(p_{0}\right) \neq 0$ and the sets

$$
\zeta^{-1}\{z ; \Im(z)=\text { const }\}, \zeta^{-1}\{z ; \Re(z)=\text { const }\}
$$

are horizontal and vertical foliations. At zeroes of $\phi d z^{2}$ a certain number of horizontal and vertical leaves resp. emanate from the singularity.

So far, the complex structure has not been changed - for a given constant $K>1$ with respect to these coordinates an affine transformation is performed with constant dilatation $K$ : On the complement of the zeroes of $\phi d z^{2}$ one defines

$$
\zeta^{\prime}=K \cdot \Re(\zeta)+i \cdot \Im(\zeta)
$$

This is in fact a complex structure of a Riemann surface with isolated points removed - a function is by definition holomorphic, provided its derivative with respect to $\overline{\zeta^{\prime}}$ vanishes. Thus the zeroes of $\phi$ are neglible and can be inserted. The existence of these points is a reason why mappings must be allowed which are not everywhere differentiable.

This construction looks like a very local argument. However, the conformal structure arises from a Riemannian metric which is of interest only up to a scalar factor. The corresponding line element is say $\left|d \zeta^{\prime}\right|^{2}$, in Teichmüller's notation

$$
d s^{2}=\lambda\left(\left|d \zeta^{2}\right|+c \cdot \Re\left(d \zeta^{2}\right)\right)
$$


where $\lambda=\left(K^{2}+1\right) / 2$ and $c=\left(K^{2}-1\right) /\left(K^{2}+1\right)$. By definition $d \zeta^{2}$ is the given holomorphic differential.

An equivalent version of (1.6) is

$$
d s^{2}=\lambda^{\prime}|d \zeta+k \overline{d \zeta}|^{2}
$$

where $k=(K-1) /(K+1)$ and $\lambda^{\prime}=(K+1) / 2$. The latter equation yields

$$
d s^{2}=\lambda^{\prime}|\phi|^{1 / 2}\left|d z+k \frac{\bar{\phi}}{|\phi|} \overline{d z}\right|^{2} .
$$

The conformal structure associated to a holomorphic quadratic differential $\phi=$ $\phi(z) d z^{2}$ and a number $k, 0<k<1$ is called a Teichmüller deformation. The corresponding quasiconformal map is set-theoretically the identity which also defines the marking.

Now the space of quadratic differentials is equipped with a norm e.g. $\|\phi\|=\int|\phi||d z|^{2}$. For any $\phi$ one sets $k:=\|\phi\|$. Then the set of all Teichmüller deformations can be identified with the open unit ball in the space of quadratic differentials. It turned out that this is exactly the Teichmüller space. Apparently it is a cell. The Teichmüller space of closed Riemann surfaces of genus $p$ is denoted by $\mathcal{T}_{p}$.

Teichmüller's theorems. Let $X_{0}$ the Riemann surface chosen as reference point, $k$ a number between 0 and 1 , and $\phi d z^{2}$ a holomorphic quadratic differential on $X_{0}$. Denote by $X_{1}$ the associated Teichmüller deformation. The Teichmüller uniqueness theorem states that the identity from $X_{0}$ to $X_{1}$ is an extremal quasiconformal map: Any diffeomorphism $f: X_{0} \rightarrow X_{1}$ which is homotopic to the identity with $K[f]<\infty$ satisfies $K[f] \geq K=(1+k) /(1-k)$. Moreover equality holds only if $f$ is the identity.

The core of Teichmüller's and any other proof is the length-area principle of Grötzsch. However, it required an investigation of the metric induced by $|\phi||d z|^{2}$, in particular the existence of geodesics.

For closed Riemann surfaces Teichmüller actually showed in [TE 1943]

Theorem. (Teichmüller's existence theorem) Any complex structure on a compact Riemann surface, viewed upon as a marked Riemann surface, is a Teichmüller deformation.

The theorem means that for any fixed Riemann surface $X_{0}$ and any diffeomorphism $g$ from $X_{0}$ to a surface $X_{1}$ there exists a Teichmüller deformation of $X_{0}$ together with a biholomorphic map to $X_{1}$ which is homotopic to $g$.

Teichmüller's considerations included an analysis of the topology of $\mathcal{T}_{p}$ related to the representation of closed Riemann surfaces in terms of Fuchsian groups.

A proof of the existence theorem is nowadays mostly worded in terms of Beltrami differentials. These are implicitely: implicitly contained in (1.8). The 
conformal structure of a Teichmüller deformation is defined by $\left|d z+k \frac{\bar{\phi}}{|\phi|} \overline{d z}\right|^{2}$ and

$$
k \frac{\bar{\phi}}{|\phi|} \frac{\overline{d z}}{d z}
$$

is called Teichmüller differential.

This is a particular case of a Beltrami differential $\mu \frac{\overline{d z}}{d z}$ (cf. sect. 2). A solution of the Beltrami equation $f_{\bar{z}}=\mu f_{z}$ is a quasiconformal map, provided $\mu$ is bounded. The Beltrami equation was studied extensively by Ahlfors and Bers. In a sense it can be used as a hinge of Teichmüller theory.

Teichmüller's theorems state that extremal quasiconformal maps always exist and correspond exactly to Teichmüller differentials.

The Moduli Space. Once the Teichmüller space is constructed the moduli space is easy to identify: Let $P$ and $Q$ be points representing marked Riemann surfaces $X_{j}$ equipped with homeomorphisms $f_{j}: X_{0} \rightarrow X_{j}, j=1,2$. Denote by $p$ the genus of $X_{0}$. Any isomorphism of $X_{1}$ and $X_{2}$ (in the abstract sense) certainly defines a homotopy class of a homoeomorphism: homeomorphism of $X_{0}$. The group $\Gamma_{p}$ of such homotopy classes is called Teichmüller modular group $\Gamma_{p}$. It can essentially be identified with the group $\operatorname{Sp}(2 p, \mathbb{Z})$ of homomorphisms of $H^{1}\left(X_{0}, \mathbb{Z}\right)$ which are compatible with the intersection pairing (cf. [GR 1961]). It acts now on $\mathcal{T}_{p}$ in a way that its orbits consist exactly of the equivalence classes of marked Riemann surfaces in the above sense, because it just operates on the markings. The quotient $\mathcal{T}_{p} / \Gamma_{p}$ is the moduli space $\mathcal{M}_{p}$. Once the Teichmüller space is equipped with a complex structure and a holomorphic family of compact Riemann surfaces, it will be clear that the action of $\Gamma_{p}$ is holomorphic (cf. sect. 2). A similar notion is used in the case of punctured Riemann surfaces.

\section{The Analytic Structure on the Teichmüller Space}

We already mentioned that in one of his last papers [TE 1944] Teichmüller considered the problem of imposing a natural complex structure on $\mathcal{T}_{p}$. He introduced the notion of an analytic family of compact Riemann surfaces ("analytische Schar Riemannscher Flächen") given by a holomorphic map of complex manifolds. He remarks that different so far not related approaches yielded the same result for the dimension of $\mathcal{T}_{p}$ seemingly by miracle — his aim was to put rather an intrinsic structure on it. He gave a description of the total space in terms of branched coverings with analytic dependence of the branch points using the Riemann-Roch theorem.

Rigorous proofs were later given by Ahlfors [AH 1960/61a] and Bers [BE 1960]. At the end one was able to understand the relationship between holomorphic families (defined by smooth, proper holomorphic maps), real analytic families of Fuchsian groups, and the space of Teichmüller differentials.

For any holomorphic family of Riemann surfaces it was natural to use the period map from the base to the generalized Siegel upper half-plane as 
essential means to introduce a complex structure on the Teichmüller space, with underlying metric and in particular topological structure being already fixed (cf. [AH 1960]).

The subsequent development was influenced by deformation theory. A. Weil had pointed out in [WE 1958] the strong relationship of Teichmüller theory and the theory of deformations of compact complex manifolds in the sense of Kodaira and Spencer. The variation of solutions of the Beltrami equation related to infinitesimal deformations provided new aspects.

\subsection{Real Analytic Theory}

Fricke Coordinates. The basic idea originating from Fricke [F-K 1926] was to represent Riemann surfaces of genus $p>1$ as quotients of the upper half plane by a discrete sub-group: subgroup of the Möbius group $\operatorname{PSL}(2, \mathbb{R})=$ $\mathrm{SL}(2, \mathbb{R}) /\{ \pm 1\}$ and to pick a set of generators, whose entries can serve as real coordinates. It was used in [AH 1960] and further developed by Keen [KE 1971]. In particular, the Teichmüller space is provided with a real analytic structure (cf. [AB 1980]).

Let $X$ be a compact Riemann surface and $\alpha_{1}, \ldots, \alpha_{2 p}$ a fixed set of generators of the fundamental group $\pi_{1}(X)$ with relations being generated by

$$
\prod_{i \text { odd }}\left[\alpha_{i}, \alpha_{i+1}\right]=1
$$

where [, ] denotes the commutator. The geometric intersection number for $i \leq j$ is

$$
\left(\alpha_{i}, \alpha_{j}\right)= \begin{cases}\delta_{i+1, j} & \text { for } i \text { odd } \\ 0 & \text { for } i \text { even }\end{cases}
$$

Such a set of generators defines in this context a marking, and the set of isomorphism classes of compact, marked Riemann surfaces of genus $p$ is called the Fricke space $\mathcal{F}_{p}$.

Theorem. The Fricke space $\mathcal{F}_{p}$ can be identified with an open subset of $\mathbb{R}^{6 g-g}$. The natural map from the Teichmüller space $\mathcal{T}_{p}$ to $\mathcal{F}_{p}$ is a homeomorphism in particular $\mathcal{T}_{p}$ carries the structure of a real analytic manifold.

In order to establish such a relationship, one assigns to the marked Riemann surface a representation $\rho: \pi_{1}(X) \rightarrow \operatorname{Aut}(H) \simeq \operatorname{PSL}(2, \mathbb{R})$, where $H$ denotes the upper half plane. The representation becomes unique under normalization. One can choose from different normalizations. One is $A_{1}(1)=1$, $A_{2}(-1)=-1$ and $A_{2}(\sqrt{-1})=\sqrt{-1}$ for $A_{j}=\rho\left(\alpha_{j}\right)$ (cf. [AH 1960]); another choice is $A_{2 p}$ with 0 as a repelling fixed point, $\infty$ as an attractive fixed point and $A_{2 p-1}(1)=1$ (cf. [AB 1980]). For those $A_{j}(z)=\left(a_{j} z+b_{j}\right) /\left(c_{j} z+d_{j}\right)$ not affected by the normalizing conditions one sets $a_{j} d_{j}-b_{j} c_{j}=1$, and $a_{j} \geq 0$, and $b_{j}>0$ if $a_{j}=0$. This yields the $6 g-6$ real so-called Fricke coordinates which are 
with respect to the latter normalization just $\left(a_{1}, b_{1}, c_{1}, \ldots, a_{2 p-2}, b_{2 p-2}, c_{2 p-2}\right)$. In fact, the equation (2.1) in terms of matrices together with the determinant condition yield the components for $j=2 g-1$ and $j=2 g$. Thus the Fricke coordinates provide an injection of the Teichmüller space into $\mathbb{R}^{6 p-6}$. By a description of transition functions a real analytic structure is imposed on $\mathcal{T}_{p}$ in [AH 1960] based upon the former, slightly different normalization. Moreover, a Fricke space of punctured Riemann surfaces can also be constructed.

The fact that the topologies of the Teichmüller and Fricke spaces match is a consequence of the Teichmüller existence theorem (with parameters) (cf. [AB 1980]).

In a direct way the introduction of a complex structure on $\mathcal{F}_{p}$ was accomplished by Kyoji Saito in [SAI 1988].

Fenchel-Nielsen Coordinates. Natural invariants of a marked Riemann surface are the lengths of closed geodesics. Also punctured Riemann surfaces are allowed. (As usual the Riemann sphere with fewer than three punctures and closed tori have to be excluded). The genus $p$ of a punctured Riemann surface is by definition the genus of its closure.

The idea is to pick sufficiently many elements $\alpha_{i}$ of the homotopy group, represent these by closed geodesics and consider the lengths $\ell\left(\alpha_{i}\right)$ with respect to the hyperbolic metric. On the other hand any such $\alpha$ corresponds to an element $\gamma$ of the Möbius group, and the quotient of the upper half plane by the cyclic group generated by $\gamma$ is an annulus or punctured disk so that $\ell(\alpha)$ can be expressed in terms of $\gamma$ as a real analytic function. L. Keen showed in [KE 1971] that a set of $6 p-6+n$ of such geodesic length functions provide real analytic coordinates on the Fricke space, i.e. these are real analytic coordinates on $\mathcal{T}_{p}$.

The approach of Fenchel and Nielsen $[\mathrm{F}-\mathrm{N}]$ is different although it also relies in a very explicit way on the hyperbolic structure of a compact Riemann surface $X$. A surface $X$ of genus $p$ with $n$ punctures is cut along a simple geodesic loop $\alpha$. A sequence of such cuts along closed geodesics $\alpha_{j}$ which do not intersect, decomposes $X$ into building blocks which are conformally equivalent to so-called "pants". These are disks with two disjoint disks removed. The removed disks may also degenerate to points in order to: to allow punctures. The number of these pants is $2 p-2+n$. As a matter of fact the conformal structure of the pants is determined by three non-negative numbers namely the geodesic lengths $\ell\left(\alpha_{j}\right)$ of the boundary components. The $\ell\left(\alpha_{j}\right)$ different from zero yield in all $3 p-3+n$ invariants.

Conversely, given a set of pants fitting in the above combinatorial pattern with (non-complete) hyperbolic metrics and matching geodesic lengths of the boundaries, the identification of boundaries turns the union into a Riemann surface $\tilde{X}$. The ambiguity of its complex structure is due to a possible displacement $\delta_{i}$ of the endpoints of the $\alpha_{i}$. This gives rise to another set of $3 p-3+p$ real numbers $\theta_{i}=2 \pi \delta_{i} / \ell\left(\alpha_{i}\right)$. In terms of the complex structure on the Riemann surface the $\theta_{i}$ are unique up to multiples of $2 \pi$. The angles $\theta_{i}$ together with the inverse numbers $\ell\left(\alpha_{j}\right)^{-1}$ define a map $\mathcal{T}_{p} \rightarrow\left(S^{1}\right)^{3 p-3+n} \times\left(\mathbb{R}^{+}\right)^{3 p-3+n}$ which is real 
analytic by a similar argument as above. Its lift $\mathcal{T}_{p} \rightarrow \mathbb{R}^{3 p-3+n} \times\left(\mathbb{R}^{+}\right)^{3 p-3+n}$ is a real analytic diffeomorphism whose components are called Fenchel-Nielsen coordinates. These have an obvious description in terms of quasiconformal mappings. The gluing with respect to an angle different from a multiple of $2 \pi$, carries geodesics transversal to a cut to broken geodesics. Multiples of $2 \pi$ induce isomorphic complex structures, i.e. elements of the modular group. The related mappings of the Riemann surface are called Dehn twists.

\subsection{The Tangent Space of $\mathcal{T}_{g}$ and its Complex Structure}

We follow Ahlfors' arguments and use his notations (cf. [AH 1961a,b]). Let $X=H / \Gamma$ be a compact Riemann surface of genus $p>1$ say and $\mu$ a Beltrami differential on $H$ which is $\Gamma$-invariant, i.e. a complex measurable function on $H$

$$
(\mu \circ A) \cdot \overline{A^{\prime}}=\mu \cdot A^{\prime}
$$

for all $A \in \Gamma$ with $\|\mu\|_{\infty}<1$. The space of $\Gamma$-invariant differentials in $L^{\infty}(H)$ is denoted by $B(\Gamma)$ and the open unit ball in it by $B(\Gamma)_{1}$.

For $\mu \in B(\Gamma)_{1}$, the normalized solution $f^{\mu}(z)$ of the Beltrami-equation

$$
\bar{\partial} f(z)=\mu(z) \partial f(z)
$$

satisfies by definition $f^{\mu}(0)=0, f^{\mu}(1)$, and $f^{\mu}(\infty)=\infty$.

The $\Gamma$-invariance of the Beltrami differential yields a transformation formula

$$
f^{\mu} \circ A=A^{\mu} \circ f^{\mu}
$$

where $A^{\mu} \in \operatorname{PSL}(2, \mathbb{R})$ can be associated to $A$ in a natural way - it yields a group $\Gamma^{\mu}$ related to the complex structure on $X$ induced by $\mu$ - a link with Fricke's approach. From the group-theoretic description in section 2.1 the Teichmüller space could be considered as the quotient $B_{1}(\Gamma) / R$, where the equivalence relation $R$ identifies Beltrami differentials $\mu_{1}$ and $\mu_{2}$, provided $A^{\mu_{1}}=A^{\mu_{2}}$ for all $A \in \Gamma$.

The Tangent Space of $\mathcal{T}_{g}$. In order to introduce local parameters on $\mathcal{T}_{g}$ in a neighborhood of the reference point $\mu$, it is sufficient to consider real analytic Beltrami differentials which are real analytic with respect to parameters. It is convenient to set $f(\nu)=f^{\nu}$. Given fixed Beltrami differentials $\nu_{1}, \ldots, \nu_{k}$, for small real $t_{1}, \ldots, t_{k}$ the function $f\left(\mu+t_{1} \nu_{1}+\ldots+t_{k} \nu_{k}\right)$ is differentiable, even real analytic, with respect to these parameters: The induced natural map which assigns to $\left(t_{1}, \ldots, t_{k}\right)$ the corresponding point in the Fricke space $\mathcal{F}_{p}$ is in fact real analytic. How could one recover the tangent space? Certainly it is necessary to introduce partial derivatives in the direction of a Beltrami differential:

$$
\dot{f}^{\mu}[\nu]:=\left.\frac{\partial}{\partial t} f(\mu+t \nu)\right|_{t=0}
$$


One may assume now that the reference structure on $X$ is the given one, i.e. $\mu=0$. Then the normalization of $f$ and (2.4) imply

$$
\bar{\partial} \dot{f}[\nu]=\nu
$$

It is obvious how to replace the real parameters by complex ones $s_{1}, \ldots, s_{k}$ and obtain from (2.7) for complex $s$

$$
\bar{\partial}\left(\left.\frac{\partial}{\partial s} f(s \nu)\right|_{s=0}\right)=\nu
$$

and

$$
\bar{\partial}\left(\left.\frac{\partial}{\partial \bar{s}} f(s \nu)\right|_{s=0}\right)=0
$$

This equation tells us that $\Phi[\nu]:=\left(\left.\frac{\partial}{\partial \bar{s}} f(s \nu)\right|_{s=0}\right)$ is holomorphic. It turned out from explicit integral representations that the range of all such $\Phi$ consists of all holomorphic functions with a certain growth condition, namely $\Phi(z)=O\left(\left(1-|z|^{2}\right)^{-2}\right)$ (with normalization $\Phi(1)=0, \Phi(-1)=0$, and $\Phi(\sqrt{-1})=0)$. Turning to $\Gamma$-invariant Beltrami differentials, one observes that the third derivatives of such functions $\phi[\nu]=\Phi[\nu]^{\prime \prime \prime}$ in fact give rise to quadratic holomorphic differentials on the Riemann surface $X$ chosen as reference point. Such holomorphic functions $\Phi$ are referred to as generalized abelian integrals. On the other hand given a quadratic holomorphic differential on $X$ with pullback $\phi$ to the unit disk $U$, the growth condition follows from the invariance under $\Gamma$. Iterated integration yields a unique function $\Phi$, vanishing at $1,-1$ and $\sqrt{-1}$ which satisfies $\Phi^{\prime \prime \prime}=\phi$. On the other hand any quadratic $\Gamma$-invariant holomorphic differential $\phi$ gives rise to an invariant Beltrami differential

$$
\nu=-\frac{1}{4}\left(1-|z|^{2}\right)^{2} \overline{\phi(z)}
$$

from which one can recover $\phi$ as $\Phi[\nu]^{\prime \prime \prime}$. This means that the space $Q(\Gamma)$ of invariant quadratic differentials can be identified with the quotient $B(\Gamma)$ of all invariant Beltrami differentials (which are say differentiable) by the subspace $N \subset B(\Gamma)$ of those, for which $\Phi$ vanishes. Its complex dimension $n=3 p-3$ is well known.

Now the tangent space can be identified with $Q(\Gamma)$ : Let a set of Beltrami differentials $\nu_{1}, \ldots, \nu_{n}$ correspond to a basis of $Q(\Gamma)$ over $\mathbb{C}$. For $s \in \mathbb{C}^{n}$ close to the origin the $f(\nu), \nu=s_{1} \nu_{1}+\ldots+s_{n} \nu_{n}$ yield generators $A_{j} \in \operatorname{Aut}(\mathrm{H})$ of $\Gamma^{\nu}$ which depend on the parameter $s$ (cf. section 2.1). These are directly related with the Fricke coordinates. It is explicitly shown that the partial derivative of all $A_{j}$ in a direction $\nu$ only vanishes, if $\dot{f}[\nu]=0$. But this means together with the above facts that the tangent map at the origin is an $\mathbb{R}$-linear isomorphism. With respect to the obvious structures of complex vector spaces it is anti-linear.

Lemma. [AH 1961a] The tangent space of the Teichmüller space at the reference point can be identified with $B(\Gamma) / N$. The latter carries a natural complex 
structure and is $\mathbb{C}$-anti-linear isomorphic to the space of holomorphic quadratic differentials.

The Complex Structure on $\mathcal{T}_{p}$ and the Universal Family. The above local, complex coordinate functions $s_{1}, \ldots, s_{n}$ are referred to as Bers coordinates. Bers showed in [BE 1960] starting from (2.5) that the choice of different reference points yields holomorphic transition functions. Bers coordinates play an essential role in geometric investigations. A main result is the existence of a holomorphic fiber space $\mathcal{X}_{p} \rightarrow \mathcal{T}_{p}$ whose fibers are the Riemann surfaces determined by the Teichmüller points. The complex structure on $\mathcal{T}_{p}$ was inherited from the (infinite dimensional) complex vector space $B(\Gamma)$ - the natural map from $B_{1}(\Gamma)$ to the Teichmüller space shall be denoted by $\mu \mapsto \theta^{\mu}$ with reference point $\theta=\theta^{0}$. Now the product $U \times \mathcal{T}_{p}$ is equipped with a holomorphic structure as follows: A complex function $F$ on a neighborhood of a point $\left(z_{0}, \theta\right)$ is by definition holomorphic, if $F\left(\theta^{\mu}, f^{\mu}\left(z_{0}\right)\right)$ is holomorphic for all $\mu$ in a neighborhood of zero in $B(\Gamma)$, a condition which descends to the quotient structure and is invariant under the choice of the reference point.

The family $\mathcal{X}=\mathcal{X}_{p} \rightarrow \mathcal{T}_{p}$ of marked Riemann surfaces has the following universal property: For any family $\mathcal{Y} \rightarrow S$ of marked Riemann surfaces (with the same topological type) the (unique) map $\phi: S \rightarrow \mathcal{T}_{p}$ such that the fibers of $s$ and $\phi(s)$ are isomorphic marked Riemann surfaces is holomorphic. Moreover it can be lifted to a map $\Phi: \mathcal{Y} \rightarrow \mathcal{T}_{p}$, whose restrictions to fibers are isomorphisms.

Action of the Modular Group. In the first section we have already seen that a change of markings yields a homeomorphism of the Teichmüller space. For $\gamma \in \Gamma$ and $t \in \mathcal{T}_{p}$, the point $t^{\prime}=\gamma(t)$ represents the Riemann surface $\mathcal{X}_{t}$ with marking induced by $\gamma$. Thus $\gamma$ induces an isomorphism $\phi_{\gamma}$ of $\mathcal{T}_{p}$ which sends $t$ to $t^{\prime}$ and lifts to an isomorphism of total spaces. In particular, the isotropy group $\Gamma_{t}$ of $t$ say acts holomorphically on the Riemann surface $\mathcal{X}_{t}$ corresponding to $t$. On the other hand automorphisms of Riemann surfaces change the marking the natural representation of $\operatorname{Aut}\left(\mathcal{X}_{t}\right)$ has $\Gamma_{t}$ as image. It is injective for $p>2$ and has kernel $\mathbb{Z}_{2}$ for $p=2$. The moduli space $\mathcal{M}_{p}$ now carries the structure of a normal complex space, since locally it can be identified with an open subset of $\mathbb{C}^{3 p-3}$ divided by the finite group of automorphisms $\Gamma_{p}$.

A Deformation Theoretic Viewpoint. In which way does this set-up: setup fit into deformation theory? One may observe that the notion $\nu$ is just a shorthand: shorthand term for

$$
\nu=\nu(z) \frac{\partial}{\partial z} \overline{d z}
$$

a ( $\bar{\partial}$-closed $)(0,1)$-form with values in the holomorphic tangent bundle of the upper half-plane. The transformation law (2.3) just means that $\nu$ descends to the Riemann surface $X$ (with respect to its complex structure). So it determines by Dolbeault cohomology a class in $H^{1}\left(X, \Theta_{X}\right)$, where $\Theta_{X}$ denotes the sheaf of holomorphic vector fields. For such a cohomology class represented by a Beltrami differential $\nu$, and a solution $f$ of the Beltrami-equation, (2.8) and (2.9) 
mean that $\nu$ represents the Kodaira-Spencer class $\rho(\partial / \partial s)$ associated to the complex tangent vector $\frac{\partial}{\partial s}$. The corresponding map $\rho: T_{0}\left(\mathcal{T}_{p}\right) \rightarrow H^{1}\left(X, \Theta_{X}\right)$ is called Kodaira-Spencer map. Now a Hodge theoretic point of view immediately implies:

Lemma. Let $X=D / \Gamma$ be a Riemann surface of genus $p>1$ equipped with the hyperbolic metric $g=\left(1-|z|^{2}\right)^{-2}|d z|^{2}$. Then the harmonic representative of a Kodaira-Spencer class is of the form

$$
\nu(z) \frac{\partial}{\partial z} \overline{d z}=\frac{\bar{\phi}}{g} \frac{\partial}{\partial z} \overline{d z}
$$

where $\phi(z) d z^{2}$ is a quadratic holomorphic differential.

Conceptually the above explicit $\mathbb{C}$-anti-linear map from the tangent space of $\mathcal{T}_{p}$ to the space of holomorphic quadratic differentials may be replaced by a duality of complex vector spaces:

For $\mu \frac{\partial}{\partial z} \overline{d z}$ and $\phi d z^{2}$, the natural pairing is

$$
\left(\mu \frac{\partial}{\partial z} \overline{d z}, \phi d z^{2}\right) \mapsto \int_{X} \mu \phi d z \overline{d z}
$$

One can verify directly that the pairing vanishes identically for all Beltrami differentials which are $\bar{\partial}$-coboundaries and that it yields a duality

$$
H^{1}\left(X, \Theta_{X}\right) \times \Gamma\left(X, \Omega_{X}^{\otimes 2}\right) \rightarrow \mathbb{C}
$$

We have seen that the harmonic representative of the Kodaira-Spencer class can be computed from the solution of the Beltrami equation.

Another approach is by the variation of the hyperbolic metrics. This is a general phenomenon for families of Kähler-Einstein manifolds, which we discuss in section 9.1 in more detail. Here we give the result which follows from [F-S 1988]:

Proposition. Let $\mathcal{X} \rightarrow S$ be a family of compact Riemann surfaces, and $g(z, s) d z \overline{d z}$ the family of hyperbolic metrics (of constant curvature -1). Let $\partial / \partial s$ be a tangent vector of $S$ at a point $s_{0} \in S$. Then the harmonic representative of $\rho(\partial / \partial s)$ is

$$
\left[-\frac{\partial}{\partial \bar{z}}\left(\frac{\partial^{2} \log g}{\partial s \partial \bar{z}} / 2 g\right)\right] \frac{\partial}{\partial z} \overline{d z}
$$

The Cotangent Space of $\mathcal{T}_{g}$. A remarkable property of the duality (2.12) is its relation with the period map $\mathcal{T}_{p} \rightarrow \mathcal{H}_{p}$, where $\mathcal{H}_{p}$ denotes the Siegel upper half plane. The second symmetric power $S^{2}\left(H^{0}(X, \Omega)\right)$ is the space of those 
quadratic differentials which come from products of differentials of the first kind on $X$. There is a natural homomorphism

$$
H^{1}\left(X_{0}, \Theta_{X}\right) \rightarrow H^{0}\left(X, \Omega^{\otimes 2}\right)^{*} \rightarrow S^{2}\left(H^{0}\left(X_{0}, \Omega\right)\right)^{*}
$$

According to [WE 1958] this composite map is exactly the derivative of the period map at the point $X$.

\section{Hyperbolicity of the Teichmüller Space}

A natural problem was to determine the automorphisms of the Teichmüller space. It was settled by the work of Royden in [RO 1971] using geometric methods for the space of closed Riemann surfaces. The result was extended by Earle and Kra to the Teichmüller space of punctured surfaces in [E-K 1974].

The main idea consisted in a characterization of biholomorphic mappings as isometries with respect to suitable metrics. It led to a comparison of the Kobayashi and Teichmüller metrics.

In section 1.1 the Teichmüller metric was defined as logarithm of the maximal dilatation of quasiconformal maps between the resp. marked Riemann surfaces. Since by Teichmüller's theorems extremal quasiconformal maps from the reference structure $X_{0}$ say to any other surface $X$ are induced by a Teichmüller differential $\mu=k \bar{\eta} /|\eta|$ for a holomorphic quadratic differential $\eta$ on $X_{0}$ and some $0<k<1$, the maximal dilatation is just $(1+k) /(1-k)$ and

$$
\tau\left(X_{0}, X\right)=(1 / 2) \log \frac{1+k}{1-k}
$$

This is for small $k$ up to second order just $k$. At least at the reference point the (real) tangent space of the Teichmüller space can be determined in terms of differentials $k^{\prime} \bar{\eta} /|\eta|$, where $k^{\prime}$ is some non-negative real number. A norm of such a Beltrami differential is just the number $k^{\prime}$.

What is the induced norm on the (complex) cotangent space of $\mathcal{T}_{p}$, if one assumes the natural pairing $(\mu, \phi)=\int_{X_{0}} \mu \phi d z \overline{d z}$ for Beltrami differentials $\mu$ and holomorphic quadratic differentials $\phi$ on $X_{0}$ ?

The result is

$$
\|\phi\|_{T}=\int_{X_{0}}|\phi| d z \overline{d z}
$$

(cf. [RO 1971]).

Lemma. The above norm \|\|$_{T}$ on the holomorphic cotangent space of the Teichmüller space is the infinitesimal form of the Teichmüller metric which is thus a Finsler metric. 
It turns out that there is more than a formal analogy of the Teichmüller distance function in terms of the excentricity $k$ to the formula for the distance of two points in the unit disk with respect to the Poincaré metric.

Given a differential $\bar{\eta} /|\eta|$, where $\eta$ is a quadratic differential as above, one may consider the differentiable map $F: D \rightarrow \mathcal{T}_{g}$ from the unit disk to the Teichmüller space, defined by $F(\zeta):=\left[X_{\zeta \cdot \mu}\right]$. Obviously the pull-back of the Teichmüller metric under $F$ equals the Poincaré metric $\rho$, and $F$ is a geodesic embedding.

This is in fact an extreme situation.

Theorem. (Royden [RO 1971]) The Teichmüller metric $\tau$ is characterized by

$$
\tau(P, Q)=\inf \rho(a, b)
$$

where the infimum is taken over all holomorphic maps $\phi: D \rightarrow \mathcal{T}_{g}$ with $\phi(a)=$ $P, \phi(b)=Q$. In particular it coincides with the Kobayashi metric.

Because of the preceding discussion it has to be shown that for any holomorphic map $\tilde{F}$ from the unit disk to $\mathcal{T}_{g}$ the pull-back $\tilde{F}^{*}(d)$ is dominated by $\rho$.

In order to show this by Ahlfors' version of the Schwarz-Pick lemma one has to construct supporting metrics of curvature at most -4 in a neighborhood of any point $p \in D$ with $\tilde{F}^{\prime}(p) \neq 0$.

One may assume $p=0$ and set $\bar{\eta} /|\eta|:=\tilde{F} \prime(p)$. To the Beltrami differential $\bar{\eta} /|\eta|$ a map $F$ can be associated according to the above construction. Then the metric $F^{*} \tau$ is up to a term $o\left(|\zeta|^{2}\right)$ supporting.

We have seen that the Teichmüller modular group $\Gamma_{g}$ acts on $\mathcal{T}_{g}$ as a group of isometries. Conversely one has the following result:

Theorem. (Royden [RO 1971]) Any biholomorphic map between domains in $\mathcal{T}_{g}$ which is an isometry with respect to $\tau$ is induced by an element of $\Gamma_{g}$.

Since any biholomorphic map of $\mathcal{T}_{g}$ is certainly an isometry with respect to the Kobayashi metric, this implies:

Corollary. Any biholomorphic map of the Teichmüller space to itself is induced by an element of the modular group $\Gamma_{g}$. For $g>2$ the groups $\operatorname{Aut}\left(\mathcal{T}_{g}\right)$ and $\Gamma_{g}$ can be identified, and $A u t\left(\mathcal{T}_{2}\right)$ is the quotient of $\Gamma_{2}$ by the group $\mathbb{Z}_{2}$.

The theorem is of purely local nature: For any two Riemann surfaces, and any $\mathbb{C}$-linear isometry of the spaces of quadratic differentials with respect to the above norm, the Riemann surfaces are isomorphic. The proof depends on a characterization of quadratic differentials $\eta$ with a zero of order $3 g-4$ by an estimate of the function $t \mapsto\left\|\eta+t \eta_{1}\right\|_{T} ; t \in \mathbb{R}$. Such differentials (if $g>2$ ) exist for any point and are unique up to a constant factor. Now, under the the: 
the 2-canonical embedding, these define the osculating hyperplanes. The given isometry of the spaces of quadratic differentials preserves this situation, and these geometric data are sufficient to show that the corresponding Riemann surfaces are isomorphic.

We add a further consequence which seems not to be contained in the literature:

Proposition. [SCH 1990][1992] Let $p>2$. Then the automorphism group of the moduli space $\mathcal{M}_{p}$ consists only of the identity.

For $p=2$ the statement does not hold, since $\mathcal{M}_{2}=\mathbb{C}^{3} / \mathbb{Z}_{5}$ has many automorphisms.

In the view of Royden's theorem it is sufficient to show that any automorphism of $\mathcal{M}_{p}$ lifts to the Teichmüller space, if $p>2$. This fact follows directly from the following lemma, since the Teichmüller space is a cell and the set of singular points of $\mathcal{M}_{p}$ is of codimension greater or equal to two.

Lemma. Let $p>2$. Then the branch locus of the map $\mathcal{T}_{p} \rightarrow \mathcal{M}_{p}$ equals the set of singularities of $\mathcal{M}_{p}$.

According to classical theorems of Chevalley, Gottschling and Prill we have to show that the modular group contains no generalized reflections. An automorphism of a complex manifold is called a generalized reflection at one of its fixed points, if its linearization has exactly one root of unity as eigenvalue with all other eigenvalues being equal to 1 .

The existence of generalized reflections in the modular group is exluded: excluded roughly as follows.

Assume that $\psi \in \Gamma_{p}$ is such an automorphism with respect to a fixed point given by some marked Riemann surface $X$. The linearized action of $\psi$ at this point is given by the action of the corresponding automorphism $\phi$ of $X$ on the space of quadratic holomorphic differentials. Consider the 2-canonical embedding of $X$ into some projective space $\mathbb{P}_{2 p-3}$. The map $\phi$ extends to some automorphism $\Phi$ of the projective space whose fixed point set consist of an isolated point $x$ and a hyperplane $H$ where $\Phi$ is a generalized reflection. The point $x$ may or may not be contained in $X$, and the intersection number of $X$ and $H$ is the degree of the 2-canonical divisor. The hyperplane $H$ intersects $X$ transversally since $\Phi$ is a reflection at these points. Now one considers the map from $X$ to its quotient by the group generated by $\phi$. A contradiction follows from the Riemann-Hurwitz formula.

\section{The Petersson-Weil Metric}




\subsection{Basic Properties}

The Petersson Scalar Product. In [PE 1949] H. Petersson had defined a scalar product for automorphic forms. For holomorphic quadratic differentials on a compact Riemann surface $X$ with hyperbolic metric $g d z \overline{d z}$ this is

$$
\left(\phi d z^{2}, \psi d z^{2}\right)_{P}=\int_{X} \frac{\phi \bar{\psi}}{g^{2}} g d z \overline{d z} .
$$

The induced norm of an element of the tangent space $H^{1}\left(X, \Theta_{X}\right)$ of the Teichmüller space with respect to the duality (2.12) can be easily computed: Let $\mu \frac{\partial}{\partial z} \overline{d z}$ be a Beltrami differential on $X$. Then the norm of its cohomology class is the supremum of $|(\mu, \psi)| /\|\psi\|_{P}$, where $\psi$ runs through all non-zero holomorphic quadratic differentials. It is convenient to evaluate (2.11) using the representative $\mu=\bar{\phi} / g$, where $\phi$ is a quadratic holomorphic differential in order to arrive at:

$$
\|\mu\|^{2}=\int_{X} \frac{|\phi|^{2}}{g} d z \overline{d z}
$$

This equation should be interpreted as follows: The Petersson inner product induces on $H^{1}\left(X, \Theta_{X}\right)$ the $L^{2}$-norm of harmonic representatives. This is the infimum of the $L^{2}$-norms over all representatives. The corresponding hermitian inner product $(,)_{P W}$ on the tangent space of $\mathcal{T}_{p}$ is called the Petersson-Weil inner product. It depends differentiably on the paramenter: parameter

Kähler Property of the Petersson-Weil Metric. In 1958 André Weil suggested the investigation of this hermitian metric on the Teichmüller space and posed the problem of its Kähler property. It was established by Ahlfors [AH 1961] using Bers coordinates (cf. section 2).

Theorem. On the Teichmüller space $\mathcal{T}_{p}, p>1$ Bers' coordinates are geodesic for the Petersson-Weil metric at the reference point. In particular this metric is Kähler.

We denote by $\omega_{P W}=g_{i \bar{\jmath}} d s^{i} d s^{\bar{\jmath}}$ the induced Kähler form on $\mathcal{T}_{p}$ which is called Petersson-Weil form.

Royden's infinitesimal description (cf. section 4) implies according to [RO 1974] a comparison of the Teichmüller metric with the Petersson-Weil metric: For any quadratic holomorphic differential $\phi d z^{2}$ the following inequality holds

$$
\int_{X}|\phi| d z \overline{d z} \leq(\operatorname{vol} X)^{1 / 2} \cdot\|\phi\|_{P}
$$

This means for the corresponding Finsler metrics on the Teichmüller space that

$$
d s_{P W} \leq[4 \pi(p-1)]^{1 / 2} d \tau,
$$

where $d \tau$ is the line element of the Teichmüller metric. (It is remarkable that an analogous inequality to (4.4) also holds on a bounded symmetric domain 
for the invariant Kähler metric and the infinitesimal Kobayashi metric on it.) The period map $\mathcal{T}_{p} \rightarrow \mathcal{H}_{p}$ is certainly distance decreasing with respect to the Kobayashi metric, a fact which can also be established on the infinitesimal level starting from (2.13). Moreover the period map is distance decreasing (up to a numerical factor) with respect to the distinguished Kähler metrics (i.e. the Petersson-Weil metric on $\mathcal{T}_{p}$ and the Bergman metric on $\mathcal{H}_{p}$.

The Petersson Weil Metric on the Moduli Space. We have seen that the Petersson-Weil metric is defined by intrinsic properties. Consider the universal family $\mathcal{X}_{p} \rightarrow \mathcal{T}_{p}$ and an arbitrary family $\mathcal{Y} \rightarrow S$ of Riemann surfaces of genus $p>1$ (provided with a marking such that it is induced by a unique holomorphic map $\phi: S \rightarrow \mathcal{T}_{p}$ ). Denote by $\Phi: \mathcal{Y} \rightarrow \mathcal{T}_{p}$ the lift of $\phi$ - its restrictions to fibers are isomorphisms: On the level of the first cohomology groups with values in the tangent bundle the pull-backs of harmonic representatives are harmonic. Thus there is a natural hermitian form (not necessarily positive definite) on $S$ which is just the pull-back of the Petersson-Weil metric. In the situation, where $\mathcal{Y} \rightarrow S$ is the Teichmüller family and $\phi$ comes from an element of the Teichmüller modular group $\Gamma_{p}$, this means that the Petersson-Weil form $\omega_{P W}$ is invariant under $\phi$. The Petersson-Weil hermitian inner product descends to the moduli space $\mathcal{M}_{p}=\mathcal{T}_{p} / \Gamma_{p}$. On a neighborhood of any point $x \in \mathcal{T}_{p}$ a $\partial \bar{\partial}$-potential of $\omega_{P W}$ can be made invariant under the isotropy group of $x$. However, the local potential descends to a function which may only be continuous. In this sense the Petersson-Weil form is defined on $\mathcal{M}_{p}$.

\subsection{The Petersson-Weil Metric for Families of Singular Riemann Surfaces}

In [BE 1974] Bers considered families of compact hyperbolic Riemann surfaces which degenerate to curves with ordinary double points. The connected components of the singular fibers, with singularities removed are called parts, and one assumes that these are all hyperbolic. Bers observed that the Poincaré metrics on the singular fibers converge in any $C^{k}$-topology to the Poincaré metrics on the parts. However, these families of metrics do not depend differentiably on the parameter. The question of the degeneracy of the Petersson-Weil metric was settled by H. Mazur in [MA 1976].

Singular Families. From the topological viewpoint a degeneration of a Riemann surface of genus $p$ consists of a contraction of closed geodesics. There are two types of such contractions - one increases the number of parts by one and leaves the sum of the genera over all parts fixed - the other decreases the genus of the corresponding part but leaves the number of parts fixed. (The genus of a part is by definition the genus of its closure).

In both cases the contraction of a cycle can be performed with a complex parameter and is compatible with the holomorphic structure. It yields a compact Riemann surface with an ordinary double point. On its (smooth) 
normalization one can distinguish the inverse image of the singularity. A compact Riemann surface together with a set of distinguished points is called a punctured Riemann surface.

The complex structure of an ordinary double point is as follows: The singularity is in terms of local coordinates $(z, w)$ with $|z|<1,|w|<1$ given by the equation $z \cdot w=0$, and a universal deformation of this singularity with a complex parameter $t,|t|<1$ is just $z \cdot w=t$. For any compact Riemann surface with an ordinary double point, this gives rise to a one-dimensional holomorphic family of compact Riemann surfaces such that the fiber over the origin is the only singular one: It is sufficient to remove a neighborhood of the double point and insert the above one-dimensional family (with biholomorphic transition functions on two pairs of annuli). On the other hand one can use Teichmüller theory of punctured Riemann surfaces. From the deformation theoretic viewpoint local families of punctured Riemann surfaces, called "stacks" in algebraic geometry, correspond to families of mappings of a finite set of say $n$ points (all equipped with the sheaf $\mathbb{C}$ ) to compact Riemann surfaces with variable complex structure. The dimension of a universal family equals $3 p-3+n$, also for genus zero or one, where $n$ must be larger than 3 or 0 resp.

A compact Riemann surface $X$ with at most ordinary double points such that its parts are hyperbolic is also called a stable curve. Its canonical sheaf can be described as follows: A section of this sheaf, pulled back to the normalization can be identified with a holomorphic 1-form on the normalization which has at most simple poles at the distinguished points and opposite residues at points which are identified under the normalization map.

A local family which is universal can be constructed as follows: The parts induce Riemann surfaces $X_{i}$ of genus $p_{i}$ with a number of $n_{i}$ punctures. These possess in particular local universal families over smooth base spaces $S_{i}$ of dimension $d_{i}=3 p_{i}-3+n_{i}$ : families $\mathcal{X}_{i} \rightarrow S_{i}$ together with sections $\sigma_{i}: S_{i} \rightarrow \mathcal{X}_{i}$. Set $S=\prod_{i} S_{i}$ and extend all families over $S$. The surface $X$ is the union of all $X_{i}$ with transversal intersections. The identification of punctures is then extended along the sections induced by $\sigma_{i}$. Finally at all singularities one applies the above insertion procedure ("opening up of nodes") relative $S$ which yields another set of $q$ local parameters, where $q$ is the number of double points. The dimension of the base is

$$
\sum_{i=1}^{r}\left(3 p_{i}-3+n_{i}\right)+q .
$$

It follows from general theory that such a family is universal for all neighboring fibers, in particular for the smooth general fiber. In fact, let a number of $q$ contractions be carried out resulting in $r$ parts $X_{i}$. Then the genus of $X$ is

$$
p=\sum_{i=1}^{r} p_{i}+q+1-r
$$

so that the dimension of the base of this family $f: \mathcal{X} \rightarrow S$ is exactly $3 p-3$. 
One may denote the coordinates which describe the deformations of singularities by $t_{j}, j=1, \ldots, q$ and those which come from deformations of punctured surfaces by $\tau_{k}, k=q+1, \ldots, 3 p-3$.

Theorem. (H. Mazur [MA 1976]) Let $S=\{(t, \tau)\}$ and $\mathcal{X} \rightarrow S$ be a family of Riemann surfaces with ordinary double points for $t_{j}=0$ for at least one $j$ and hyperbolic parts as above. Then the following estimates for the components $g_{i \bar{j}}$ of the Petersson-Weil metric tensor hold:

(i) There exist constants $0<a<A$ and a neighborhood of $(0,0)$, where

$$
\frac{-a}{\left|t_{i}\right|^{2}\left(\log \left|t_{i}\right|\right)^{3}} \leq g_{i \bar{\imath}} \leq \frac{-A}{\left|t_{i}\right|^{2}\left(\log \left|t_{i}\right|\right)^{3}} \quad \text { for } \quad i \leq q
$$

(ii)

(iii)

$$
\begin{aligned}
\left|g_{i \bar{\jmath}}(t, \tau)\right|= & O\left(\frac{1}{\left|t_{i}\right|\left|t_{j}\right|\left(\log \left|t_{i}\right|\right)^{3}\left(\log \left|t_{j}\right|\right)^{3}}\right) \\
& \text { as } \quad(t, \tau) \rightarrow(0,0) \quad \text { for } \quad i, j \leq q, i \neq j
\end{aligned}
$$

(iv)

$$
\lim _{(t, \tau) \rightarrow(0,0)} g_{i \bar{\jmath}}(t, \tau)=g_{i \bar{\jmath}}(0,0) \quad \text { if } \quad i, j>q
$$

$$
\left|g_{i \bar{\jmath}}(t, \tau)\right|=O\left(\frac{-1}{\left|t_{i}\right|\left(\log \left|t_{i}\right|\right)^{3}}\right) \quad \text { as } \quad(t, \tau) \rightarrow(0,0) \quad \text { if } \quad i \leq q, j>q .
$$

These estimates have several consequences. The Petersson-Weil metric is not complete. In transversal direction to the divisor $t_{1} \cdot \ldots \cdot t_{q}=0$ it degenerates, whereas restricted to the "parallel" direction it converges to the Petersson-Weil metric on families of punctured Riemann surfaces. Moreover, one can read off the equations that the volume of the moduli space $\mathcal{M}_{p}$ is finite.

In fact Mazur treats components

$$
g^{i \bar{\jmath}}=\int \phi_{i}(t, \tau) \overline{\phi_{j}(t, \tau)} g(t, \tau) d z d \bar{z}
$$

first, where the $\phi_{i}$ are holomorphic sections of the relative 2-canonical bundles $\Omega_{\mathcal{X} / S}^{\otimes 2}$ which are dual to the holomorphic vector fields $\partial / \partial t_{i}$ and $\partial / \partial \tau_{j}$ on $S$. The sections $\phi_{i}$ originate from a basis of $\Omega_{\mathcal{X} / S}(S)$, where the first $q$ sections related to $\partial / \partial t_{i}$ contain the factor $t_{i}$.

The specific estimates of (4.5) are due to the degeneration of both the family of hyperbolic metrics and of these sections. These relative quadratic differentials are meromorphic with at most double poles in the $z_{i}$-direction (in terms of local coordinates $\left(z_{i}, w_{i}, t_{i}\right)$ near the singularities). These coordinates are sometimes called "pinching coordinates". The relative metric tensor $g(t, \tau)$ can be estimated using the embedding of the annuli $\left\{\left(z_{i}, t_{i}\right) ;\left|t_{i}\right|<\right.$ 
$\left.\left|z_{i}\right|<1\right\}$ equipped with hyperbolic metrics as follows: For small $\left|t_{i}\right|$, the annuli $\left\{\left(z_{i}, t_{i}\right) ;\left|t_{i}\right|<\left|z_{i}\right|<1\right\}$ are holomorphically embedded into the fibers of the family $\mathcal{X} \rightarrow S$. This means that the hyperbolic metrics $h\left(z_{i}, t_{i}\right)$ dominate the restrictions of the Poincaré metrics $g(z, t, \tau)$ of the fibers. Furthermore the maximum principle for elliptic differential equations yields that the supremum of $h / g$ for fixed parameters is attained on the boundary of the respective disks. For $\left|z_{i}\right|=\delta<1$ or $\left|w_{i}\right|=\delta<1$ by Bers' result one has uniform convergence of the metric tensors $g$ to the hyperbolic metric on the respective parts in all $C^{k}$-topologies.

An alternative approach can be based solely on estimates of the metric tensor, since by (2.13) the tensor of the Petersson-Weil metric can be computed in terms of the variation of the hyperbolic metric on the family.

\section{The Curvature of the Petersson-Weil Metric}

\subsection{Ahlfors' Results}

Shortly after the Kähler property of the Petersson-Weil metric was established, Ahlfors showed in [AH 1961b]

Theorem. The Ricci curvature, the holomorphic sectional curvature and the scalar curvature of the Petersson-Weil metric are negative.

His proof is a continuation of the computations leading to the Kähler property involving an integral formula for the curvature tensor.

\subsection{Bounds of the Curvature}

Two questions remained open for more than ten years: The negativity of the sectional curvature and the existence of a negative upper bound for the holomorphic sectional curvature.

In [RO 1974] Royden conjectured a precise such upper bound. It was established by Wolpert in [WO 1986] and Tromba [TR 1986]:

\section{Theorem.}

(i) The holomorphic sectional and Ricci curvatures of $\omega_{P W}$ on $\mathcal{T}_{p}$ for $p>1$ are bounded from above by $\frac{-1}{2 \pi(p-1)}$.

(ii) The sectional curvature of $\omega_{P W}$ is negative.

In [WO 1986] such results are also attributed independently to Royden. This theorem is a direct consequence of an explicit formula for the curvature tensor. We refer here to Wolpert's approach. Let $X$ be a compact Riemann surface of genus $p>1$. Denote by $\left\{\mu_{\alpha} ; \alpha=1, \ldots, 3 p-3\right\}$ a basis of the vector 
space of harmonic Beltrami differentials on $X$ corresponding to a set of tangent vectors $\left\{\partial / \partial s_{\alpha} ; \alpha=1, \ldots, 3 p-3\right\}$. The product of such a differential with a conjugate one is a differentiable function, and we have seen that the components of the Petersson-Weil form

$$
\omega_{P W}=\sqrt{-1} g_{\alpha, \bar{\beta}} d z^{\alpha} \wedge d z^{\bar{\beta}}
$$

are in terms of the basis

$$
g_{\alpha \bar{\beta}}=\int_{X} \mu_{\alpha} \overline{\mu_{\beta}} g d z d \bar{z}
$$

Denote by $D$ the real laplacian on $L^{2}$-functions on $X$ (with non-positive spectrum). Then the inverse $(D-2)^{-1}$ exists and is a compact integral operator.

Theorem. (Wolpert [WO 1986], Tromba [TR 1986]) The curvature tensor of the Petersson-Weil metric equals

$$
\begin{aligned}
R_{\alpha \bar{\beta} \gamma \bar{\delta}}= & -2 \int_{X}(D-2)^{-1}\left(\mu_{\alpha} \overline{\mu_{\beta}}\right)\left(\mu_{\gamma} \overline{\mu_{\delta}}\right) d z d \bar{z} \\
& -2 \int_{X}(D-2)^{-1}\left(\mu_{\alpha} \overline{\mu_{\delta}}\right)\left(\mu_{\gamma} \overline{\mu_{\beta}}\right) g d z d \bar{z} .
\end{aligned}
$$

We indicate, how to derive the various estimates of the curvatures of $\omega_{P W}$ from (5.2): One sets $\Delta=-2(D-2)^{-1}$. This is an integral operator with a positive kernel, a fact which implies that for all $L^{2}$-functions $\phi$ and $\psi$ the inequality

$$
|\Delta(\phi \psi)| \leq\left|\Delta \phi^{2}\right|^{1 / 2}\left|\Delta \psi^{2}\right|^{1 / 2}
$$

holds. The negativity of the sectional curvature follows from the explicit formula (5.2) by means of (5.3) and the Hölder inequality.

Given an $L^{2}$-function $\phi$ on $X$, an eigenfunction expansion of $\phi$ with respect to the laplacian $D$ implies:

$$
\int_{X} \Delta(\phi) \bar{\phi} g d z d \bar{z} \geq\left|\int_{X} \phi g d z d \bar{z}\right|^{2} / \int_{X} g d z d \bar{z}
$$

The estimates of the holomorphic sectional and Ricci curvatures are based on (5.2) and (5.4).

The following condition goes beyond negative sectional curvature.

Definition. A Kähler manifold is of strongly negative curvature in the sense of Siu, if its Riemann tensor satisfies

$$
R_{\alpha \bar{\beta} \gamma \bar{\delta}}\left(A^{\alpha} \overline{B^{\beta}}-C^{\alpha} \overline{D^{\beta}}\right)\left(\overline{A^{\delta}} B^{\gamma}-\overline{C^{\delta}} D^{\gamma}\right) \geq 0
$$


for all complex vectors $A^{\alpha}, B^{\beta}, C^{\gamma}, D^{\delta}$, and if equality holds only for $A^{\alpha} \overline{B^{\beta}}=$ $C^{\alpha} \overline{D^{\beta}}$ for all $\alpha$ and $\beta$.

Proposition. [SCH 1986] The Petersson-Weil metric on $\mathcal{T}_{p}$ for $p>1$ has strongly negative curvature in the sense of Siu.

We note that this condition is not satisfied for the invariant metric on bounded symmetric domains (cf. [SI 1986]).

\subsection{The Curvature of the Petersson-Weil Metric for Singular Families}

In section 5.2 we discussed the asymptotics of the Petersson-Weil metric for families with singular fibers. We assume again that the parts of the singular fibers are hyperbolic and use the above notations.

Theorem. [SCH 1986] Let $S=\{(t, \tau)\}$ and $\mathcal{X} \rightarrow S$ be a family of Riemann surfaces with ordinary double points and hyperbolic parts. Then

(i) The absolute values of the sectional, Ricci-: Ricci and scalar curvature are of type $O\left(-\sum_{i=1}^{q} \log \left|t_{i}\right|\right)$.

(ii) the Ricci tensor of the Petersson-Weil metric satisfies

$\left|R_{i, \bar{j}}\right|= \begin{cases}O\left(\frac{1}{\left|t_{i}\right| \cdot \log \left|t_{i}\right| \cdot\left|t_{j}\right| \cdot \log \left|t_{j}\right|} \cdot \min \left(\frac{1}{\log ^{2}\left|t_{i}\right|}, \frac{1}{\left.\log ^{2} \mid t_{j}\right) \mid}\right)\right. & \text { if } i, j \leq q \text { and } i \neq j \\ O\left(\frac{1}{\left|t_{i}\right|^{2} \cdot \log ^{2}\left|t_{i}\right|}\right) & \text { if } i=j \leq q \\ O\left(\frac{1}{\left|t_{i}\right|^{2} \cdot \log ^{3}\left|t_{i}\right|}\right) & \text { if } i \leq q \text { and } j>q \\ O(1) & \text { if } i, j>q\end{cases}$

In [SCH 1986] also estimates for the Christoffel symbols and the curvature tensor of the Petersson-Weil metric are given.

In order to prove such estimates, one considers partial derivatives of $g^{i \bar{j}}$ according to (4.5) using the setup of section 4.2. The holomorphic vector fields $\partial / \partial t_{i}$ and $\partial / \partial \tau_{k}$ are lifted to $C^{\infty}$ vector fields $v_{i}$ on $\mathcal{X}$ which are with respect to pinching coordinates around the singularties: singularities of the form $\partial / \partial t_{i}$ and $\partial / \partial \tau_{k}$ resp. Thus it is sufficient to estimate the respective Lie derivatives of the tensors, which occur inside the integral (4.5). The holomorphic quadratic differentials $\phi_{i}$, whose poles are exactly known don't cause any difficulty. The main point is to estimate the derivatives of the metric tensor $g$.

\section{Lemma.}

$$
\begin{aligned}
\left|L_{v_{i}}(g)\right| / g & =O\left(\frac{-1}{\left|t_{i}\right| \log \left|t_{i}\right|}\right), \quad i=1, \ldots, q \\
\left|L_{v_{\bar{\jmath}}} L_{v_{i}}(g)\right| / g & =O\left(\frac{1}{\left|t_{i}\right|\left|t_{j}\right| \log \left|t_{i}\right| \log \left|t_{j}\right|}\right) ; \quad i, j=1, \ldots q
\end{aligned}
$$


Here we need a global argument. One intruduces: introduces an auxiliary metric $h\left(z, t_{i} \tau_{k}\right)$ which is around the singularities related to the hyperbolic metric on annuli and punctured discs resp. The term $\log (g / h)$ and its derivatives with respect to the vector fields $v_{i}$ satisfy global elliptic equations, from which the lemma follows.

These methods are sufficient for the estimates of the curvature tensor of the Petersson-Weil metric except for: except $R_{i \bar{\imath} i \bar{\imath}}$. One observes that the harmonic Beltrami differentials $\mu_{i}$ corresponding to $\partial / \partial t_{i}$ and $\partial / \partial \tau_{k}$ can be expressed in terms of the dual basis $\left\{\phi_{i}\right\}$ and the Petersson-Weil metric by $\mu_{i}=\sum_{j} g_{i \bar{\jmath}} \cdot \overline{\phi_{j}} / g$. Thus (4.2) can be used to show

$$
\left|R_{i \bar{i} i \bar{\imath}}\right|=O\left(\frac{-1}{\left|t_{i}\right| \log ^{5}\left|t_{i}\right|}\right) \quad \text { for } \quad i \leq q .
$$

This method yields weaker estimates for the other terms.

A consequence of the above estimates is that

$$
-\int_{\mathcal{M}_{p}} \operatorname{Ric}\left(\omega_{P W}\right) \wedge \omega_{P W}^{3 p-4}<\infty
$$

\section{Harmonic Maps and Teichmüller Space}

Description of $\mathcal{T}_{p}$ in Terms of Hyperbolic Metrics. Another way how to phrase the classification problem of complex structures on a compact surface $X$ of genus $p>1$ is to consider all Riemannian metrics of constant negative curvature -1 say up to diffeomorphisms. This is due to the fact that any such complex structure gives rise to a unique hyperbolic metric on one hand and that on the other hand any Riemannian metric determines a conformal i.e. complex structure. If (for fixed genus) $\mathcal{M}_{-1}$ denotes the space of such metrics and $\mathcal{D}_{0}$ the group of diffeomorphisms homotopic to the identity, then the quotient space $\mathcal{M}_{-1} / \mathcal{D}_{0}$ can be identified with the Teichmüller space $\mathcal{T}_{p}$. Here we do not specify the analytic structure on $\mathcal{M}_{-1}$ and $\mathcal{D}_{0}$ resp. - one may consider $C^{\infty}$-metrics and -diffeomorphisms or Sobolev spaces of $H^{k}$-metrics and $H^{k+1}$-diffeomorphisms. Such quotients have been considered for Riemannian manifolds of arbitrary dimension by Berger and Ebin, who prove a slice theorem in [B-E 1969], and by Fischer and Tromba in [F-T 1984].

Harmonic Maps of Riemann Surfaces. The idea of using harmonic maps to investigate Teichmüller space originates from Gerstenhaber and Rauch (cf. [G$\mathrm{R}$ 1954] and [RE 1985]). We refer here to the approach by Fischer-Tromba [F-T 1984a,b], Tromba [1986/7], Wolf [W 1989], and Jost [JO 1989][1990]. According to a theorem of Schoen and Yau [S-Y 1978] for any two metrics $g$ and $\gamma$ in $\mathcal{M}_{-1}$ there exists a unique harmonic map $v:(X, g) \rightarrow(X, \gamma)$ which is homotopic to the identity. This fact also allows the following interpretation: For 
a fixed hyperbolic (reference) metric $g$ on $X$ one can choose in any $\mathcal{D}_{0}$-orbit a unique hyperbolic metric $\tilde{\gamma}$ such that the identity $(X, g) \rightarrow(X, \tilde{\gamma})$ is harmonic.

The link between classical Teichmüller theory and the approach via harmonic maps is that harmonic maps are closely related to quadratic holomorphic differentials. Let $g$ and $\gamma$ be Riemannian metrics on $X$ of constant negative curvature -1 , and $z, w$ holomorphic coordinate functions on $X$ related to the metrics $g$ and $\gamma$ - by abuse of notation we write $g(z) d z \overline{d z}$ and $\gamma(w) d w \overline{d w}$. The energy density of such a mapping $z \mapsto w(z)$ of class $C^{1}$ say is

$$
e(w)=\mathcal{H}+\mathcal{L},
$$

where

$$
\mathcal{H}=\gamma(w(z))\left|w_{z}\right|^{2} / g(z) \text { and } \mathcal{L}=\gamma(w(z))\left|w_{\bar{z}}\right|^{2} / g(z)
$$

The engergy: energy functional on the space of such mappings $w$ is

$$
E(w)=\int_{X} e(z) g(z) d z \overline{d z} .
$$

It only depends on the conformal structure of the domain, as well as the Euler-Lagrange equation for its critical points, the harmonic maps:

$$
w_{z \bar{z}}+\left(\frac{\gamma_{w}}{\gamma} \circ w\right) \cdot w_{z} w_{\bar{z}}=0
$$

An immediate consequence of (6.2) is the following characterization of the decomposition of the pull back of the metric tensor $\gamma$ under $w$ :

$$
w^{*}(\gamma) d w \overline{d w}=\phi d z^{2}+e(w) \cdot g d z \overline{d z}+\bar{\phi} \overline{d z}^{2}
$$

where the quadratic differential

$$
\phi d z^{2}:=g w_{z} \bar{w}_{z} d z^{2}
$$

is holomorphic.

Fixing the hyperbolic metric $g$ on the domain (and its complex structure), one has altogether: all together a natural map from the Teichmüller space to the space of holomorphic quadratic differentials:

$$
\Phi: \mathcal{T}_{g} \rightarrow H\left(X, \Omega_{X}^{\otimes 2}\right)
$$

In fact the map $\Phi$ provides global coordinates on the Teichmüller space.

\section{Theorem.}

(i) (Sampson [SA 1978]): The map $\Phi$ is injective.

(ii) (M. Wolf [W 1989]): The map $\Phi$ is surjective.

Since $\log \mathcal{H}$ satisfies an elliptic equation, the injectivity of $\Phi$ is reduced to the maximum principle, and since $\Phi$ is continous: continuous due to regularity 
theorems, the surjectivity follows from the properness of $\Phi$ which is ultimately the properness of the energy functional.

What is the relation with classical Teichmüller theory, in particular Bers coordinates? We do not expect to find in this way global holomorphic coordinates on the Teichmüller space, but the statement of the theorem suggests to identify: identifying maybe the holomorphic tangent space of $\mathcal{T}_{p}$.

The theorem implies that for a basis $\phi_{1}, \ldots, \phi_{3 p-3}, \quad t=\left(t_{1}, \ldots t_{3 p-3}\right) \in$ $\mathbb{C}^{3 p-3}$ and $\phi(t)=\sum_{j} t_{j} \phi_{j}$ there exist hyperbolic metrics $\gamma(t)$ and harmonic maps $z \mapsto w(z, t)$, satisfying (6.4). Since $w(z, 0)=z$, one can easily compute the derivative with respect a the complex parameter $t_{j}$ of the $(0,2)$-component of $w^{*}(\gamma)$ at $t=0$. On one hand it is certainly $\bar{\phi}_{j}$, on the other hand must equal: it must equal $\left.g \cdot\left(\partial^{2} w / \partial t_{j} \partial \bar{z}\right)\right|_{t=0}$ : The derivative of the associated Beltrami differential (with respect to the complex parameter $t_{j}$ ) is just $\bar{\phi}_{j} / g$. This means that the map $\Phi$ of the above theorem supplies an identification of the complex tangent space of $\mathcal{T}_{p}$ at the reference point given by $(X, g)$ with the space of quadratic holomorphic differentials. When restricted to the first infinitesimal neighborhood of the distinguished fiber the induced differentiable family of compact Riemann surfaces over $H^{0}\left(X, \Omega_{X}^{\otimes 2}\right)$ can in fact be identified with to the Teichmüller family equipped with the local holomorphic Bers coordinates.

The Petersson-Weil Metric. In the above approach of Wolf, the hyperbolic metric on the domain is fixed - previously Tromba had used the opposite standpoint to describe the Petersson-Weil metric by means of harmonic maps. In both set-ups: setups the total energy of harmonic maps gives rise to a real function on the Teichmüller space.

Theorem. (Tromba [TR 1987]) The energy function on $\mathcal{T}_{p}$ is a $\partial \bar{\partial}$-potential for the Petersson-Weil metric at the reference point.

A further application is the computation of the curvature of the PeterssonWeil metric (cf. section 5).

Rigidity of $\overline{\mathcal{M}}_{p}$. Based on the classical theory of harmonic maps in Riemannian geometry and a $\partial \bar{\partial}$-Bochner-type formula Siu showed in [SI 1980] a strong rigidity theorem for compact Kähler manifolds $M$ of strongly negative curvature. It states that any compact Kähler manifold of the same homotopy type must be either biholomorphic or conjugate biholomorphic to $M$. The strong negativity of the Petersson-Weil metric was verified in [SCH 1986] and under restrictions, related to the branching of the action of the Teichmüller modular group any harmonic map from $\overline{\mathcal{M}}_{p}$ to itself is either holomorphic or antiholomorphic. A strong rigidity theorem for $\overline{\mathcal{M}}_{p}$ was shown by Jost and Yau in [J-Y 1987]. 


\section{The Compactified Moduli Space}

\subsection{Properties of $\overline{\mathcal{M}}_{p}$}

Local Description. The quasi-projectivity of $\mathcal{M}_{p}$ was established by different methods. Baily [BA 1962] used the Jacobi-map: Jacobi map, and $\Theta$-series. Baily and Borel constructed a compactification $\widetilde{\mathcal{M}_{p}}$ by means of the period map related to the compactification of the moduli space of polarized abelian varieties in [B-B 1966]. Here the complement of the moduli space in $\widetilde{\mathcal{M}_{p}}$ is of codimension two. Another approach in terms of Kleinian groups and horocyclic coordinates is described in Kra's article [KR 1989][1990]).

Although a compactification $\overline{\mathcal{M}}_{p}$ is a priori not uniquely defined, there is a natural choice, where the compactifying divisor $\overline{\mathcal{M}}_{p} \backslash \mathcal{M}_{p}$ has a geometric interpretation. We shall refer here to the method due to Mumford [MU 1977] and Knudsen [KN 1983b], who construct $\overline{\mathcal{M}}_{p}$ as a compact projective variety with at most quotient singularities. In section 4.2 we essentially discussed $\overline{\mathcal{M}}_{p}$ from the analytic viewpoint, namely local universal families of compact (connected) Riemann surfaces with at most ordinary double points, whose parts are hyperbolic, i.e. stable curves.

The stability condition guarantees that only a finite number of cycles may be contracted yielding terminal singularities. These correspond to points, where the largest possible number of locally irreducible components of the compactifying divisor intersect. Moreover there exist only finitely many choices of contractions which lead to terminal singularities. In terms of Fenchel-Nielsen coordinates (cf. section 2.1) one can see that for geodesic length functions $\ell(\alpha)$ tending to zero, one arrives at an ordinary double point. This description suggests that the divisor $\mathcal{D}=\overline{\mathcal{M}}_{p} \backslash \mathcal{M}_{p}$ is a union $\mathcal{D}=\mathcal{D}_{0} \cup \ldots \mathcal{D}_{[p / 2]}$, where the components $\mathcal{D}_{j}$ can be described as follows: The generic points of $\mathcal{D}_{0}$ correspond to complex curves of genus $p-1$ and 2 punctures, and for $\mathcal{D}_{j} ; j>0$ these are curves consisting of two components of genus $j$ and $p-j$ resp. with one puncture each.

As for the intersection of components of $\mathcal{D}$ one may consider now a (local, universal) family $\mathcal{C} \rightarrow S$ of stable curves, where $S \subset \mathbb{C}^{3 p-3}$ like in section 5.2 and 5.3 and $p>1$ is the genus of the general fiber. Let $t_{j}$ be coordinate functions on the base such that the divisor of singular fibers equals $V\left(t_{1} \cdot \ldots \cdot t_{q}\right)$. We have seen that over the sets $V\left(t_{j_{1}}, \ldots, t_{j_{r}}\right) \backslash \bigcup_{k \neq j_{i}} V\left(t_{k}\right)$ the curve $\mathcal{C}$ induces a locally universal family of Riemann surfaces with $r$ punctures. The subset of $\overline{\mathcal{M}}_{p}$ corresponding to $S$ can be identified with the quotient of $S$ by a finite group of automorphisms.

Ample Line Bundles on $\overline{\mathcal{M}}_{p}$. In [MU 1977], Mumford gives explicitely: explicitly an ample line bundle on $\overline{\mathcal{M}}_{p}$. The sheaf of relative holomorphic 1-forms $\Omega_{\mathcal{X} / S}^{1}$ on a family of stable curves has been defined in section 5.2. For the local description one may consider a one-dimensional family $f: \mathcal{C} \rightarrow T$ given locally by $V(z \cdot w-t) \rightarrow T ; \quad(z, w, t) \mapsto t$. Then the relative dualizing sheaf $\omega_{\mathcal{C} / T}$ is the invertible sheaf generated by a differential $\zeta$ which equals $d z / z$ for $z \neq 0$ 
and $-d w / w$ for $w \neq 0$. Let $\mathbf{m}_{0} \subset \mathcal{O}_{T}$ be the maximal ideal sheaf for $0 \in T$. Then the sheaf $\Omega_{\mathcal{C} / T}^{1}$ can be identified with the subsheaf $\mathbf{m}_{0} \cdot \omega_{\mathcal{C} / T}$. Like in the non-singular case sections of $\omega_{C}^{\otimes e}$ for $e \geq 3$ provide an embedding of a stable curve into a projective space of dimension $\nu-1=(2 e-1)(p-1)-1$, where the degree of the image is $d=2 e(p-1)$. The Hilbert polynomial $P(n)=\chi\left(C, \omega_{C}^{\otimes n}\right)$ equals $d n-(g-1)$. The space of all such $e$-canonical curves embedded as above is parametrized by a locally closed, smooth subscheme $H$ of the Hilbert scheme $H i l b_{\nu-1}^{P}$, i.e we are given a family of embedded curves

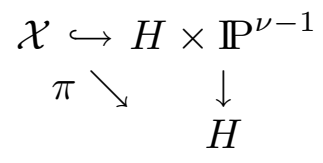

Any isomorphism of stable curves is induced by a linear map of the ambient space, and this means a natural action of $G=P G L(\nu-1, \mathbb{C})$ on $H$. The term "stable" refers to this group action - it means that all orbits are closed and all stabilizer groups $G_{x}$ are finite. Geometric invariant theory provides the existence of $\overline{\mathcal{M}}_{p}$ in the sense described so far.

The main point is now to associate to a given relatively ample line bundle $\mathcal{L}$ on $\mathcal{X}$ a line bundle on the base $H$ which is close to ample. At least generically the direct images of $\mathcal{L}^{\otimes n}$ are locally free and the highest exterior powers (on the base $H$ ) are natural candidates. Moreover, for a construction based on intrinsic objects everything should descend to $\overline{\mathcal{M}}_{p}$, yielding ultimately an ample line bundle on the compactified moduli space.

We give a few details, since the construction makes an impact on the the: the theory of moduli of higher dimensional manifolds. The natural choice for $\mathcal{L}$ is $\omega_{\mathcal{X} / H}$. Its powers give rise to the direct image sheaves $\pi_{*}\left(\omega_{\mathcal{X} / H}^{\otimes n}\right)$. The first direct images vanish except for $n=1$, where the result is the structure sheaf of $H$. Thus the first images are locally free (by the comparison theorem). One denotes

$$
\lambda_{n}=\Lambda^{\max }\left(\pi_{*} \omega_{\mathcal{X} / H}^{\otimes n}\right)=\operatorname{det}\left(\mathbf{R}^{\bullet} \pi_{*}\left(\omega_{\mathcal{X} / \mathrm{H}}^{\otimes \mathrm{n}}\right)\right) ; \lambda:=\lambda_{1}
$$

It turns out that the choice of the power $n$ is immaterial. The line bundles $\lambda_{n}$ can be gotten hold of by computing their Chern classes. Let $\pi_{!}: K(\mathcal{X}) \rightarrow K(H)$ denote the morphism of Grothendieck groups of coherent analytic sheaves, then $c_{1}\left(\lambda_{n}\right)=c_{1}\left(\pi_{!} \omega_{\mathcal{X} / H}^{\otimes n}\right)$ more or less by definition. The latter term is accessible from the generalized Riemann-Roch formula - it is just the degree 2 component of the direct image of the product of the character of the relative dualizing sheaf and the Todd character of the sheaf of regular relative 1 -forms:

$$
c_{1}\left(\lambda_{n}\right)=\left[\pi_{*}\left(\operatorname{Ch}\left(\omega_{\mathcal{X} / H}^{\otimes n}\right) \cdot \operatorname{Td}\left(\Omega_{\mathcal{X} / H}^{1}\right)\right)\right]_{2}
$$

This expression is expanded as usual. At this place the above relationship between $\Omega_{\mathcal{X} / H}^{1}$ and $\omega_{\mathcal{X} / H}$ implies that the quotient of these is the structure 
sheaf $\mathcal{O}_{\operatorname{Sing}(\mathcal{C})}$ of the singular locus. Its first Chern class vanishes, and the second is represented by the associated 2 -codimansional cycle $[\operatorname{Sing}(\mathcal{C})]$. The evaluation of the right-hand side of (7.2) yields the the: the following theorem, since the Picard group of $\overline{\mathcal{M}}_{p}$ is torsion free.

Theorem. (Mumford [MU 1977])

(i)

$$
\lambda_{n}=\lambda^{\otimes\left(6 n^{2}-6 n+1\right)} \otimes\left(\delta^{-1}\right)^{\otimes \frac{n(n-1)}{2}},
$$

where $\delta=[\mathcal{D}]$.

(ii) The bundles $\lambda_{n}$ descend to the compactified moduli space $\overline{\mathcal{M}}_{p}$.

(iii) The bundle

$$
\lambda^{\otimes b} \otimes\left(\delta^{-1}\right)^{\otimes a}
$$

is ample on $\overline{\mathcal{M}}_{p}$ if $a>0$ and $b>11.2 a$, and not ample if $a \leq 0$ or $b \leq 11 a$.

The last statement contains the fact that $\lambda_{2}=\operatorname{det}\left(\pi_{*}\left(\omega_{\mathcal{X} / H}^{\otimes 2}\right)\right)=\lambda^{\otimes 12} \otimes \delta^{-1}$ is ample.

There is a relation with Baily's result. Let $\mathcal{A}_{p, 1}$ the moduli space of principally polarized abelian varieties of dimension $p$ and $J: \mathcal{M}_{p} \rightarrow \mathcal{A}_{p, 1}$ the map which sends a compact Riemann surface to its Jacobian. Denote by $\Theta: \mathcal{A}_{p, 1} \rightarrow \mathbb{P}^{N}$ the realization via $\Theta$-functions. Then

Theorem. (Mumford [MU 1977]) The map $J \circ \Theta$ extends to $\theta: \overline{\mathcal{M}}_{p} \rightarrow \mathbb{P}^{N}$ so that for some $m$,

$$
\theta^{*}\left(\mathcal{O}_{\mathbb{P}^{N}}(1)\right)=\lambda^{m}
$$

Again using the generalized Riemann-Roch formula, Harris and Mumford computed in [H-M 1982] the canonical class of the compactified moduli space:

$$
K_{\overline{\mathcal{M}}_{p}} \equiv 13 \lambda-2 \delta_{0}-3 \delta_{1}-2 \delta_{2}-\ldots-2 \delta_{\left[\frac{p}{2}\right]}
$$

where the $\delta_{j}$ correspond to the components of the compactifiying divisor.

Theorem. (Harris-Mumford [H-M 1982], Harris [HA 1985]) The moduli space $\mathcal{M}_{p}$ is of general type, if the genus is odd and at least 25, or if the genus is even and at least 40 . 


\subsection{The Petersson-Weil Class on $\overline{\mathcal{M}}_{p}$ as Chern Class of a Positive Line Bundle}

The unification of algebraic geometry and the analytic approach to Teichmüller theory was achieved by Wolpert's work.

In his results the Petersson-Weil form plays a central role. Its class in $H^{2}\left(\mathcal{M}_{p}, \mathbb{R}\right)$ generates this space and $\left(1 / 2 \pi^{2}\right)\left[\omega_{P W}\right]$ is rational and extends to an element of $H^{2}\left(\overline{\mathcal{M}}_{p}, \mathbb{Q}\right)$. It corresponds essentially to a line bundle, and the Petersson-Weil form extends as a $(1,1)$-current which is the Chern form of a continuous hermitian metric on this bundle which is known from algebraic geometry. A smoothing of the metric shows the positivity of this line bundle on $\overline{\mathcal{M}}_{p}$.

A link with Mumford's results is the following fiber-integral formula. One considers the Teichmüller family where the fibers are equipped with the hyperbolic metrics. These turn the relative anti-canonical bundle into a hermitian line bundle. Denote by $c_{1}\left(\mathcal{X}_{p} / \mathcal{T}_{p}, g\right)$ its Chern form. Its second power is a (2,2)-form whose fiber integral is a (1,1)-form on the Teichmüller space. (By functoriality the construction is compatible with base change and everything descends to the moduli space).

Theorem. (Wolpert [WO 1986])

$$
\frac{1}{2 \pi^{2}} \omega_{P W}=\int_{\mathcal{X}_{p} / \mathcal{T}_{p}} c_{1}\left(\mathcal{X}_{p} / \mathcal{T}_{p}, g\right)^{2}
$$

This formula can be viewed upon as an extension of (7.2) to Chern forms rather than classes emphasizing the importance of the Petersson-Weil form.

In the sequel we shall describe the results mentioned above in more detail.

Extension of the Petersson-Weil Class to the Compactified Moduli Space. The device, how to construct an extension $\left[\omega_{P W}\right] \in H^{2}\left(\overline{\mathcal{M}}_{p}, \mathbb{R}\right)$ is to change the differentiable structure near the compactifying divisor $\mathcal{D}$. The symplectic structure on the Teichmüller space can be expressed in an amazingly simple way in terms of Fenchel-Nielsen coordinates:

Theorem. (Wolpert [WO 1985a]) Let $0<\ell_{j}<\infty$ and $-\infty<t_{j}<\infty$ for $j=1, \ldots, 3 p-3$ be Fenchel-Nielsen coordinates on $\mathcal{T}_{p}$. The the: The PeterssonWeil form equals:

$$
\omega_{P W}=-\sum_{j} d \tau_{j} \wedge d \ell_{j}
$$

The proof of the duality formula $\omega_{P W}\left(\partial / \partial \tau_{\alpha}, \quad\right)=-d \ell_{\alpha}$ is based on a very detailed investigation of Fenchel-Nielssen vector fields and hyperbolic 
geometry, in particular relations for the "twist derivatives" $\left(\partial / \partial \tau_{\alpha}\right)\left(\ell_{\beta}\right)$ and $\left(\partial^{2} / \partial \tau_{\alpha} \partial \tau_{\beta}\right)\left(\ell_{\gamma}\right)$ using Petersson series.

A rather direct consequence of (7.3) is that the Petersson-Weil form (pushed down to the moduli space) possesses a differentiable extension $\omega^{F N}$ (in the sense of $\mathrm{V}$-manifolds) to $\overline{\mathcal{M}}_{p}$.

On the other hand, the Petersson-Weil form is singular near the compactifying divisor with respect to the complex structure of the moduli space. The exact estimates are quoted in section 5.2. These imply immediately that $\omega_{P W}$ is of class $L^{2}$ - the extension of $\omega_{P W}$ to $\overline{\mathcal{M}}_{p}$ as a $(1,1)$-current with respect to the complex structure is denoted by $\omega^{C}$. Moreover Mazur's estimates imply that the integral of the product of $\omega_{P W}$ with the exterior derivative of a differentiable $(6 p-9)$-form with compact support in a neighborhood of some point of $\mathcal{D}$ vanishes. In particular (by de Rham cohomology on $\mathrm{V}$-manifolds) it induces a linear functional on $H^{6 p-8}\left(\overline{\mathcal{M}}_{p}, \mathbb{R}\right)$, by Poincaré duality this gives an element of $H^{2}\left(\overline{\mathcal{M}}_{p}, \mathbb{R}\right)$.

Theorem. (Wolpert [WO 1985a]) The cohomology classes of $\omega^{F N}$ and $\omega^{C}$ in $H^{2}\left(\overline{\mathcal{M}}_{p}, \mathbb{R}\right)$ are equal.

The proof of this fact requires certain prerequesits: prerequesites: One is a combination of Čech-: Čech and Dolbeault cohomology theory. The other is the fact that the identity $i: \overline{\mathcal{M}}_{p}^{F N} \rightarrow \overline{\mathcal{M}}_{p}^{C}$ of the compactified moduli space equipped with the Fenchel-Nielsen and complex structures resp. is Lipschitz continuous (as opposed to $i^{-1}$ which is not even Hölder continuous). The Lipschitz condition is finally reduced to the boundedness of the Fenchel-Nielsen coordinate vector fields with respect to $L^{2}$ - and $L^{1}$-norms and estimates of $\left\|d s_{k}\right\|$, where $s_{k}$ are holomorphic coordinate functions near the compactifying divisor.

Rationality of the Petersson-Weil Class. Now two methods became available for investigating the Petersson-Weil class. The smooth 2-form may be integrated over 2-cycles or paired with $(n-2)$-forms; the Kähler form $\omega^{C}$ reflects the complex geometry of $\overline{\mathcal{M}}_{p}, \omega^{C}$ is positive on all complex tangent planes.

The rationality of $\left(1 / \pi^{2}\right)\left[\omega_{P W}\right]$ (on the compactified moduli space) is based on a computation of the rational homology of $\overline{\mathcal{M}}_{p}$. According to a result of Harer [HA 1983] the group $H_{2}\left(\mathcal{M}_{p}, \mathbb{Q}\right)$ is of rank one for $p>2$. Wolpert considers in [WO 1983] the components $\mathcal{D}_{0}, \ldots, \mathcal{D}_{[p / 2]}$ of the compactifying divisor $\mathcal{D}$. He can show that the real first cohomology classes $\left[\omega_{P W}\right] \in H_{6 p-8}\left(\overline{\mathcal{M}}_{p}, \mathbb{R}\right)$ and those induced by $\mathcal{D}_{0}, \ldots, \mathcal{D}_{[p / 2]}$ constitute a basis by considerations of the intersection pairing - the rank of $H_{2}\left(\overline{\mathcal{M}_{p}}, \mathbb{Q}\right)$ is $2+[p / 2]$. The components of $\mathcal{D}$ together with the line bundle $\lambda$ are known to form a set of generators of $\operatorname{Pic}\left(\overline{\mathcal{M}}_{p}\right) \otimes \mathbb{Q}$. A computation of the intersection pairing implies that $\left(1 / \pi^{2}\right)\left[\omega_{P W}\right]$ is in fact rational - it replaces the class of the Hodge line bundle in the above basis of $H^{2}\left(\overline{\mathcal{M}}_{p}, \mathbb{Q}\right)$. Here the Fenchel-Nielsen description (6.1) of the extended Petersson-Weil class is essential. In particular $\left[\omega_{P W}\right]$ is the 
Chern class of a line bundle. The final step of this program is to show that the Petersson-Weil form is induced by a hermitian metric on an ample line bundle which is continuous over $\overline{\mathcal{M}}_{p}$ and of class $C^{\infty}$ over $\mathcal{M}_{P}$.

A Local Continuous Kähler Potential for $\omega_{P W}$. As above we use the setup of section 5.2. A local Kähler potential $F$ is a solution of the equation $\sqrt{-1} \partial \bar{\partial} H=$ $\omega_{P W}$

The estimates of the Petersson-Weil metric on the cotangent bundle, in particular its boundedness, guarantee that $g_{i \bar{\jmath}}$ possesses a smooth Hermitian metric as minorant. Thus the associated $(1,1)$-current $\left[\omega_{P W}\right]$ on $\overline{\mathcal{M}}_{p}$ is positive. The $\partial \bar{\partial}$-Poincaré lemma yields locally a strictly plurisubharmonic potential $P$ in the sense of currents.

Any potential $H$ satisfies $\Delta H=\operatorname{Trace}(\sqrt{-1} \partial \bar{\partial} H)=\operatorname{Trace}\left(\omega_{P W}\right)=: h$, where $h$ is comparable to $\left|t_{1}\right|^{-2}\left(\log 1 /\left|t_{1}\right|\right)^{-3}+\ldots+\left|t_{q}\right|^{-2}\left(\log 1 /\left|t_{q}\right|\right)^{-3}$.

A (weak) solution $H$ of the Laplace equation $\Delta H=h$ is a convolution with the Green's function $G$. It can be verified, using the asymptotics of $h$ and $G$ that $H$ is continuous, and from the regularity theorem for solutions of the homogeneous equation it follows that the distribution $P$ can be represented by a contionous: continuous plurisubharmonic function.

A Continuous Hermitian Metric. So far all computations are related to local families of compact Riemann surfaces (with singularities). The base of such a family yields a coordinate neighborhood for the V-structure of $\overline{\mathcal{M}}_{p}$. The notions of hermitian metrics and Chern forms are carried over to to: to V-manifolds. Let $\lambda$ be a holomorphic line bundle on $\overline{\mathcal{M}}_{p}$, whose real Chern class is an integer multiple of $\left(1 / \pi^{2}\right)\left[\omega_{P W}\right]$. The local continuous Kähler potentials give rise to a continuous hermitian metric on $\lambda$ with the prescribed curvature form.

Smoothing of the Kähler Current and Projective Embedding of $\overline{\mathcal{M}}_{p}$. Although the Petersson-Weil class on the compactified moduli space is related to a positive current and up to a numerical factor to the Chern class of a line bundle $\lambda$, this bundle need not be a priori positive. This is accomplished by Richberg's theorem. His result is applied to show the following general fact: Let $L$ be a line bundle on a complex manifold an $h$ a continuous hermitian metric which is locally given by strongly plurisuperharmonic functions. Then there exists a hermitian metric of class $C^{\infty}$ and strictly positive Chern form which is cohomologous to the Chern form of $f$ as current. In particular, $L$ is a positive line bundle.

In this way $\lambda$ becomes a positive holomorphic line bundle on the $\mathrm{V}$ manifold $\overline{\mathcal{M}}_{p}$, i.e. with respect to local uniformizing systems the hermitian metric is smooth with positive curvature. Since positive line bundles on Vmanifolds yield embeddings into projective spaces (cf. [BA 1957]), the compactified moduli space $\overline{\mathcal{M}}_{p}$ has a projective embedding determined by the positive line bundle associated to the Petersson-Weil current $\left(1 / \pi^{2}\right) \omega_{P W}$. 
Chapter B

\section{Moduli Spaces of Compact Kähler Manifolds}

\section{Existence Theorems for Moduli Spaces of Polarized Kähler Manifolds}

Notions. What is the proper analogue of the Teichmüller space and the moduli space of compact Riemann surfaces for compact complex manifolds of arbitrary dimension?

A moduli space also called "coarse moduli space" is a complex space which classifies compact complex manifolds up to isomorphism. Its points correspond to such isomorphism classes and its complex structure reflects the variation of the complex structure on a fixed differentiable manifold in a holomorphic family. This idea will be made more precise. (As it stands, it gives the notion of a moduli space in the reduced category, but at the little expense of technical extensions everything can be generalized to the non-reduced case).

Set-theoretically the points of a moduli space $\mathcal{M}$ consist of isomorphism classes $[X]$ of compact complex manifolds $X$. A holomorphic family of compact complex manifolds $\mathcal{X}_{s} ; s \in S$, parametrized by a (reduced) complex space $S$, is given by a proper, smooth, holomorphic map $f: \mathcal{X} \rightarrow S$ of complex spaces with connected fibers, such that $\mathcal{X}_{s}=f^{-1}(s)$ for all $s$. Thus one can assign to any such family $f$ the natural map

$$
\phi_{f}: S \rightarrow \mathcal{M}
$$

which relates a point $s \in S$ to the isomorphism class $\left[\mathcal{X}_{s}\right]$ of its fiber.

The above set of isomorphism classes is called a moduli space, if it carries the structure of a complex space such that all maps of the type $\phi_{f}$ are holomorphic. (For non-reduced moduli spaces one has simply to require the existence of such maps, compatible with base change, whose reduction has the above property). This condition determines the holomorphic structure of a moduli space uniquely. The general problem is not well-posed: There exist families $f$ of compact manifolds over a one-dimensional base, such that all fibers but one are isomorphic to each other - the map $\phi_{f}$ has to be constant, unless one introduces non-hausdorff moduli spaces. Certain families of Hopf surfaces or Hirzebruch surfaces have this property. The former may stand for non-kähler manifolds, the latter for ruled manifolds. Furthermore one knows from the moduli theory of abelian varieties that one has to assign a polarization. 
The notion of a polarization deserves a short discussion: In the algebraic situation it is related to a model i.e. a realization of variety $X$ in a projective space. The embedding is defined by a couple of sections of a line bundle $\mathcal{L}$ on $X$, in fact, $\mathcal{L}$ is just the restriction of the hyperplane section bundle of the respective projective space. A polarization in the algebraic sense is induced by a line bundle, a power of which has this property. For Riemann surfaces of genus larger than one or manifolds with negative first Chern class it is given in an implicit way: These are called canonically polarized, since the canonical bundle, i.e. the bundle of holomorphic differential forms of highest degree, yields a polarization.

The interpretation of a polarization as an integer-valued Kähler class supposes the correct notion - a polarized Kähler manifold $\left(X, \lambda_{X}\right)$ is by definition equipped with a Kähler class $\lambda \in H^{2}(X, \mathbb{R})$, and an isomorphism of polarized manifolds by definition is a biholomorphic map which carries one polarization to the other.

Moduli of abelian varieties, the period map and the Riemann relations initiated the theory of variations of Hodge stucture with implications for the theory of moduli of K3-surfaces, symplectic and unitary manifolds (cf. [TO 1988]).

Our aim here is to develop a general theory of moduli spaces of Kähler manifolds, based on deformation theory.

Methods. We shortly review basic notions of deformation theory.

Definition. Let $X$ be a compact complex manifold and $\left(S, s_{0}\right)$ a complex space with a distinguished point $s_{0}$.

$A$ deformation of $X$ over $\left(S, s_{0}\right)$ consists of a family $\mathcal{X} \rightarrow S$ of compact complex manifolds together with an isomorphism $X \stackrel{\sim}{\rightarrow} \mathcal{X}_{s_{0}}$.

A holomorphic map $\left(R, r_{0}\right) \rightarrow\left(S, s_{0}\right)$ induces a deformation of $X$ over $R$ which is defined by the family $\mathcal{X}_{R}:=\mathcal{X} \times{ }_{S} R \rightarrow R$ together with the induced isomorphism between $X$ and the fiber of $r_{0}$. This is called base-change.

An isomorphism of deformations is an isomorphism of families which induces the identity on $X$.

$A$ deformation of a manifold $X$ is called complete, if it generates all deformations by means of base-change up to isomorphism after replacing the base spaces by neighborhoods of the distinguished points, if necessary.

A deformation is called effective if the derivative of a base change in the sense above at the distinguished point is uniquely determined - complete and effective deformations are called versal.

$\mathbf{A}$ a: A universal deformation is a complete deformation, where the above base-change itself is uniquely determined.

The main theorem of deformation theory states the existence of versal deformations of compact complex spaces.

In the sense of a general theory of moduli spaces based on deformation theory an initial result for polarized Kähler manifolds was: 
Theorem. [SCH 1983b] There exists the moduli space of polarized Kähler manifolds with vanishing first real Chern class.

In order to construct a moduli space for manifolds of a certain type or with additional structure one has to perform the following steps which shall be explained below more precisely:

- construction of versal (complete and effective) deformations (of polarized manifolds)

- union of quotients with identification of those points which correspond to isomorphic fibers

At this stage the set of isomorphism classes carries a natural topology. For a complex structure the following is essential:

- universality of versal deformations of manifolds of the type under consideration.

- action of the automorphism group of the central fiber on base such that the orbits consist of exactly the equivalent points.

The automorphism group of a polarized manifold $\left(X, \lambda_{X}\right)$ is a complex Lie group which contains the identity component $\operatorname{Aut}^{0}(X)$, since the action of this group on $H^{2}(X, \mathbb{R})$ is trivial.

The notion of a family of polarized Kähler manifolds is very natural. The Kodaira-Spencer stability theorem means that neighboring fibers of a Kähler manifold are again Kähler - however, the Kähler class only depends differentiably on the parameter. Thus it is reasonable to define a polarization of a family $f: \mathcal{X} \rightarrow S$ as a (holomorphic) section $\lambda_{\mathcal{X} / S}$ of the direct image $\mathbf{R}^{1} f_{*} \Omega_{\mathcal{X} / S}$, whose restrictions $\lambda_{s}:=\lambda \mid \mathcal{X}_{s}$ are polarizations of the fibers. Heuristically such a real-valued holomorphic function ought to be constant: in fact it determines a section of the locally constant sheaf $\mathbf{R}^{2} f_{*} \mathbb{R}$, whose restrictions to arbitrary fibers are Kähler classes. The converse is also true (up to some extra condition for non-reduced bases spaces).

On the other hand there is the notion of a Kähler morphism $f: \mathcal{X} \rightarrow S$ which is by definition a proper smooth map of complex spaces equipped with a locally $\partial \bar{\partial}$-exact real $(1,1)$-form, whose restriction to any fiber is a Kähler form. (It comes from a Čech-1-cochain of differentiable functions which induces a 2cocycle with values in the sheaf of pluri-harmonic functions $\mathcal{H}$ on $\mathcal{X}$.

It may be remarkable that any family $f$ of polarized Kähler manifolds is a Kähler morphism, even if $S$ is singular. This follows from a very simple argument, whereas the Kodaira-Spencer stability theorem involves forth order elliptic equations. ${ }^{1}$

$\overline{1}$ Consider on $\mathcal{X}$ the short exact sequence $0 \rightarrow \mathbb{R} \rightarrow \mathcal{O} \rightarrow \mathcal{H} \rightarrow 0$, where $\mathcal{H}$ denotes the sheaf of pluri-harmonic functions. Assume $S$ to be Stein and reduced such that $\mathbf{R}^{2} f_{*} \mathbb{R}$ is constant over $S$. The polarization determines an element of $H^{2}(\mathcal{X}, \mathbb{R})$, whose image in $H^{2}(\mathcal{X}, \mathcal{H})$ vanishes, since all restrictions to fibers do, $\left(\mathbf{R}^{2} f_{*} \mathcal{O}\right.$ is locally free). Thus the polarization comes from a 1-cocycle in $\mathcal{H}$ which can be 
The introduction of polarized families suggests that the corresponding deformation theory makes sense; with respect to any holomorphic map $R \rightarrow S$ the pull-back $\mathcal{X}_{R}=\mathcal{X} \times{ }_{S} R \rightarrow R$ carries a natural polarization. On the other hand, for any proper, smooth morphism $\tilde{f}: \widetilde{\mathcal{X}} \rightarrow \tilde{S}$ and a polarization on a distinguished fiber $\mathcal{X}_{s_{0}}$, there is a maximal subgerm of $\left(S, s_{0}\right)$ over which the polarization can be extended (uniquely). In such a way the following existence theorem can be proved (cf. [SCH 1983a,1984]):

Proposition. Let $\left(X, \lambda_{X}\right)$ be a polarized Kähler manifold. Then there exists a versal deformation.

This first step in the necessary program for the construction of a moduli space could be done with no further assumption. It ensures that $\mathcal{M}$ carries a natural topology.

In order to get hold of isomorphic fibers in a family one may look out for a classifying space for isomorphisms between two given families and their pullback with respect to a base change: Let $\left(\mathcal{X} \rightarrow S, \lambda_{\mathcal{X} / S}\right)$ and $\left(\mathcal{Y} \rightarrow S, \lambda_{\mathcal{Y} / S}\right)$ be such families. The relevant theorem also exists in the polarized case (cf. [SCH 1984] also [SCH1983b]):

Proposition. There exists a holomorphic map $I \rightarrow S$ and a universal isomorphism $\Phi: \mathcal{X}_{I} \rightarrow \mathcal{Y}_{I}$ over $I$ of polarized families. One sets $I=\operatorname{Isom}_{S}^{\lambda}(\mathcal{X}, \mathcal{Y})$.

The fibers $I_{s}$ of $I=\operatorname{Isom}_{S}^{\lambda}(\mathcal{X}, \mathcal{Y}) \rightarrow S$ can be identified with the sets of isomorphisms between $\mathcal{X}_{s}$ and $\mathcal{Y}_{s}$; thus these are, if not empty, isomorphic to the groups of automorphisms of such a fiber - in particular the fibers are smooth.

We have the following general criterion for the existence of a moduli space.

Theorem. [SCH 1983b, 1984] Let $\mathcal{K}$ be a collection of compact polarized manifolds which possess versal deformations (in $\mathcal{K})$. Then there exists a moduli space for $\mathcal{K}$, if for any two families $\left(\mathcal{X} \rightarrow S, \lambda_{\mathcal{X} / S}\right)$ and $\left(\mathcal{Y} \rightarrow S, \lambda_{\mathcal{Y} / S}\right)$ of polarized manifolds the morphism $I=\operatorname{Isom}_{S}^{\lambda}(\mathcal{X}, \mathcal{Y}) \rightarrow S$ is proper.

The properness of the above holomorphic map just means that for any sequence $s_{\nu}$ in $S$ converging to some $s_{0} \in S$ and any isomorphisms $\phi_{\nu}$ of the polarized fibers $\mathcal{X}_{s_{\nu}}$ and $\mathcal{Y}_{s_{\nu}}$ there exists a sub-sequence converging to an isomorphism of the fibers of $s_{0}$.

As it stands the criterion is just the condition for the topological space $\mathcal{M}$ to be hausdorff. However, it automatically guarantees that the further analytic steps can be performed.

written as the coboundary of differentiable functions giving rise to an exact $(1,1)$ form. This differs from a given Kähler form on a distinguished fiber by $\partial \bar{\partial}$ of a differentiable function which can be extended to all of $\mathcal{X}$. 
Its proof is based on purely deformation theoretic arguments - the only assumptions are the existence of versal deformations, the existence of a classifying space of isomorphisms of holomorphic families, and the above properness. ${ }^{2}$ In this situation we have the following lemma:

Let $\left(X, \lambda_{X}\right)$ be a polarized Kähler manifold in $\mathcal{K}$ and $S$ the base of its versal deformation. Denote by $\sim$ the equivalence relation on $S$ induced by the isomorphy of fibers.

Lemma. [SCH 1983b, 1984] Under the assumption of the theorem, any versal deformation of $X$ is universal.

After replacing $S$ by a neighborhood of the distinguished point the finite group $G=\operatorname{Aut}^{\lambda}(X) / \operatorname{Aut}^{0}(X)$ acts on $S$, and the quotient $S / \sim$ can be naturally identified with $S / G$.

We indicate very shortly the method of the proof: Let the versal deformation of $X$ be noted in terms of a cartesian diagram:

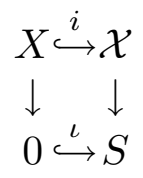

One can check directly that the graph $\Gamma \subset S \times S$ of $\sim$ is the image of the (proper) canonical map $\kappa: I=\operatorname{Isom}_{S \times S}^{\lambda}(\mathcal{X} \times S, S \times \mathcal{X}) \rightarrow S \times S$. Any $\phi \in \operatorname{Aut}^{\lambda}(X)$ gives rise to another deformation of $X$. By the versality of the given deformation there exists a cartesian diagram

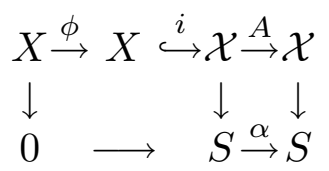

where $A \circ i \circ \phi=i$. We assign to any $\phi$ such a base change morphism $\alpha=\alpha(\phi)$ which is a priori not uniquely determined by $\phi$ and the diagram.

The map $(\alpha, i d): S \rightarrow S \times S$ has values in $\Gamma$, and from the versality of the given deformation one can derive that the projection from $S \times S$ onto the second component, restricted to $\Gamma$ is finite around $\left(s_{0}, s_{0}\right)$, where $s_{0}=\iota(0)$. In particular, there exist only finitely many choices for $\alpha$. The assumption implies that $\operatorname{Aut}^{\lambda}(X)$ is compact, in particular abelian. Let $\operatorname{Aut}^{\varepsilon}(X) \subset \operatorname{Aut}^{\lambda}(X)$ denote the subgroup of automorphisms which are extendable to the whole family over some

2 The general version is as follows: Let $\mathbf{A s}$ and $\mathbf{A g}$ be the categories of complex spaces and germs of complex spaces resp. Let $p: \mathbf{F} \rightarrow \mathbf{A n}$ be a fibered groupoid and $q: \mathbf{G} \rightarrow \mathbf{A g}$ the induced groupoid. Assume that any object $a_{0}$ of $\mathbf{G}$ such that $q\left(a_{0}\right)$ is the reduced point posseses: possesses a versal deformation $a$ and that versality is an open condition. Then there exists a moduli space for $p: \mathbf{F} \rightarrow \mathbf{A g}$ under the condition: For any complex space $S$ and any $b, c$ in $\mathbf{F}$ with $p(b)=p(c)=S$ the functor $\operatorname{Isom}(b, c): \mathbf{A s} \rightarrow$ Sets is representable by a space $\iota: I \rightarrow S$, where $\iota$ is proper. 
neighborhood of $s_{0}$. Let $\phi, \psi \in \operatorname{Aut}^{\lambda}(X)$. Then $\alpha(\phi)=\alpha(\psi)$ implies that the deformations induced by $\phi$ and $\psi$ are isomorphic, i.e. $\phi \circ \psi^{-1} \in \operatorname{Aut}^{\varepsilon}(X)$. Thus: Thus, the quotient set $\operatorname{Aut}^{\lambda}(X) / \operatorname{Aut}^{\varepsilon}(X)$ is finite. In particular $\operatorname{Aut}^{\varepsilon}(X) \subset$ $\operatorname{Aut}^{\lambda}(X)$ which implies $\operatorname{Aut}^{0}(X) \subset \operatorname{Aut}^{\varepsilon}(X)$, i.e. the dimension of the automorphism groups $\operatorname{Aut}\left(\mathcal{X}_{s}\right)$ is constant: we are dealing in fact with a universal deformation. Now the assignment $\alpha \mapsto \phi$ is unique and gives rise to a representation $\rho: G=\operatorname{Aut}^{\lambda}(X) / \operatorname{Aut}^{\varepsilon}(X) \rightarrow \operatorname{Aut}\left(S, s_{0}\right)$. If we let act $G$ on $S \times S$ trivially on the first component and by $\rho$ on the second, then we can see that the orbits of points $(s, s)$ are contained in $\Gamma$.

We claim that on the other hand, the irreducible components $\Gamma_{j}$ of $\Gamma$ through $\left(s_{0}, s_{0}\right)$ are of the form $\{(s, g \cdot s) ; s \in S\}$ for some $g \in G$ : The embedding of a component $\Gamma_{j}$ into $S \times S$ followed by either projection yields two families with isomorphic fibers, i.e. the image of $I \rightarrow S \times S$ contains $\Gamma_{j}$, and since this map is proper with smooth fibers, it possesses a section over $\Gamma_{j}$ - both families over $\Gamma_{j}$ are isomorphic. The existence of the desired group element $g \in G$ follows from deformation theory.

Altogether: All together, both quotients $S / \sim$ and $S / G$ are homeomorphic and one can see that the complex structure on the quotient, induced by the finite group $G$, has the necessary properties.

Remark. The universality of the versal deformation follows already from the compactness of $\operatorname{Aut}^{0}(X)$.

To see this, we need $\operatorname{Aut}^{0}(X) \subset \operatorname{Aut}^{\epsilon}(X)$ in the above argument: The compactness of $\operatorname{Aut}^{0}(X)$ implies that a neighborhood of the "point" $\phi \in I$ in $I$ is mapped in a proper way to a neighborhood of $\left(s_{0}, s_{0}\right) \in S \times S$ (cf. [F-S 1988b]).

Results. How could one verify the assumptions of the criterion? The following idea proved crucial: represent the polarization by a distinguished Kähler form such that any biholomorphic map between polarized manifolds exactly corresponds to an isometry. This is already sufficient - the properness of the classifying space of isomorphisms over the base follows now from the classical theorems of van Dantzig-van der Waerden and Myers-Steenrod.

In order to apply this argument to prove an existence theorem for the moduli space of polarized manifolds with vanishing first Chern class, one can take the unique Ricci flat metrics (representing the polarization) according to Yau's solution of the Calabi problem as such distinguished metrics. This method using Kähler-Einstein metrics also yields an analytic approach to the moduli space of canonically polarized manifolds. Furthermore one can combine it with the proof of the Matsusaka-Mumford theorem to show the existence of the moduli space of those polarized Kähler manifolds, for which some power of the canonincal bundle is generated by global sections (cf. [SCH 1984]).

Based upon the methods in [SCH 1983b], using the Kähler analogue of the Matsusaka-Mumford theorem in [FU 1981] the general theorem was proved: 
Theorem. [FU 1984, SCH 1984] There exists the moduli space of polarized nonuniruled Kähler manifolds.

(Uniruled manifolds possess many rational curves - by definition these manifolds are the images of $\mathbb{P}_{1}$-bundle spaces under meromorphic maps which are not constant on the fibers of the bundle).

One may note that the topology of the moduli space is countable, if the underlying differentiable manifold $M$ say is fixed and equipped with a class $\lambda \in H^{2}(M, \mathbb{R})$ (cf. [F-S 1988a]).

Another situation, where the criterion is applicable, are polarized Hodge manifolds. These are compact complex manifolds together with the isomorphism class of an ample line bundle ${ }^{3}$ (cf. [F-S 1988b]). The result is the existence theorem of the moduli space of non-uniruled Hodge manifolds. ${ }^{4}$

\section{Moduli Spaces of Kähler-Einstein Manifolds}

\subsection{The Generalized Petersson-Weil Metric}

Definitions. So far, we already emphasized the close relationship between complex and metric structure on Kähler manifolds as related to the existence of a moduli space.

Let $X$ be a complex Kähler manifold with Kähler form $\omega_{X}$ and Ricci form $\operatorname{Ric}\left(\omega_{X}\right)$. The Kähler form is called Kähler-Einstein, if

$$
\operatorname{Ric}\left(\omega_{X}\right)=k \cdot \omega_{X}
$$

for some real number $k$ which can be normalized to $-1,0$ or 1 . (The first Chern form equals $\left.(1 / 2 \pi) \operatorname{Ric}\left(\omega_{X}\right)\right)$.

In terms of local holomorphic coordinates $\left(z^{1}, \ldots, z^{n}\right)$ we have

$$
\omega_{X}=\sqrt{-1} g_{\alpha, \bar{\beta}} d z^{\alpha} \wedge d z^{\bar{\beta}}
$$

where $g_{\alpha, \bar{\beta}}$ denotes the metric tensor. One denotes $g=\operatorname{det} g_{\alpha, \bar{\beta}}$ so that the induced volume form equals $\left(\omega_{X}\right)^{n}=2^{n} n ! g d v, d v$ the euklidian: euclidean volume element. Then $\operatorname{Ric}\left(\omega_{X}\right)=-\sqrt{-1} \partial \bar{\partial} \log g$ which equals $\sqrt{-1} R_{\alpha, \bar{\beta}} d z^{\alpha} \wedge d z^{\bar{\beta}}$ in terms of the Ricci tensor $R_{\alpha \bar{\beta}}$.

In order to describe the generalized Petersson-Weil metric we have to define the corresponding hermitian form on the tangent spaces of universal deformations. This form is compatible with base-change so that it descends to the moduli space. Let $\left(X, g_{\alpha \bar{\beta}}\right)$ be a compact Kähler-Einstein manifold of constant

3 It is convenient to use isomorphism classes in order to avoid $\mathbb{C}^{*}$-parts in the isomorphism space.

4 A further generalization is the existence of the moduli space of non-uniruled manifolds $X$ with refined Kähler class (these are elements of $H^{1}(X, \mathcal{H})$, induced by Kähler forms). 
(non-positive) curvature. Let $\left(S, s_{0}\right)$ be the base of a universal deformation. By means of the Kodaira-Spencer map $\rho$, the tangent space of $S$ at $s_{0}$ is identified with $H^{1}\left(X, \Theta_{X}\right)$.

This cohomology group is just the space of obstructions against a holomorphic lift of a tangent vector of the base to the total space (to be precise: to a holomorphic vector field on the infinitesimal neighborhood of the fiber which projects to the given tangent vector). Thus: Thus, in terms of Dolbeault cohomology $\rho$ is defined in the following way: let $\left(z^{1}, \ldots, z^{n}\right)$ be local holomorphic coordinates of the fiber and $\left(s^{1}, \ldots, s^{k}\right)$ holomorphic coordinates of $S$ with respect to a smooth ambient space such that $(z, s)$ can be taken as local coordinates on $\mathcal{X}$. A tangent vector on $S$ at $s_{0}$ different from zero is the of: of the form $\partial / \partial s$ for some coordinate function $s$. Now take a differentiable lift of the form $b^{\alpha}(z) \partial / \partial z^{\alpha}+\partial / \partial s$. Then $B_{\bar{\beta}}^{\alpha} \frac{\partial}{\partial z^{\alpha}} d z^{\bar{\beta}}$ represents the Kodaira-Spencer class, where $B_{\bar{\beta}}^{\alpha}=\partial b^{\alpha} / \partial z^{\bar{\beta}}$.

Let $u \in T_{s_{0}}(S)$ be a tangent vector and

$$
\eta=A_{\bar{\beta}}^{\alpha} \frac{\partial}{\partial z^{\alpha}} d z^{\bar{\beta}}
$$

be the harmonic representative of $\rho(u)$ with respect to the Kähler metric $g_{\alpha \bar{\beta}}$ in terms of local holomorphic coordinates $\left(z^{1}, \ldots, z^{n}\right)$.

Definition. The Petersson-Weil inner product on $T_{s_{0}}$ is given by the following norm

$$
\|u\|_{P W}^{2}:=\int_{X}|\eta|^{2} g d v=\int_{X} A_{\bar{\beta}}^{\alpha} \bar{A}_{\gamma}^{\bar{\delta}} g^{\alpha \bar{\delta}} g_{\gamma \bar{\beta}} g d v
$$

where gdv denotes the volume element with respect to the given metric.

The harmonicity of $\eta$ is just the following equation:

$$
0=\bar{\partial}^{*} \eta=g^{\bar{\beta} \gamma} A_{\bar{\beta} ; \gamma}^{\alpha} \frac{\partial}{\partial z^{\alpha}} .
$$

(The semi-colon: semicolon denotes covariant derivatives).

First the Kähler property of the generalized Petersson-Weil metric on nonsingular base spaces was introduced by Koiso in [KO 1983] starting from the point of Riemannian geometry and Einstein manifolds. A technical assumption for the case of Ricci-flat metrics could be replaced by the assumption of a polarized family (for this observation cf. [SCH 1985]).

Siu computed explicitely: explicitly the curvature of the generalized PeterssonWeil metric in [SI 1986].

The verification of the Kähler property heavily depended on the fact that the above harmonic representatives of the Kodaira Spencer classes give rise to symmetric 2-tensors:

$$
A_{\bar{\beta} \bar{\delta}}=A_{\bar{\delta} \bar{\beta}}
$$

where $A_{\bar{\beta} \bar{\delta}}=g_{\alpha \bar{\beta}} A_{\bar{\delta}}^{\alpha}$. 
The tensor $A_{\bar{\beta} \bar{\delta}}$ describes in fact the variation of the Kähler-Einstein metrics on the fibers of our universal family in the direction of the tangent vector $u$. This shall be made more precise below.

Some results of [F-S 1988b] shall now be displayed.

A Fiber-Integral Formula. Wolpert's formula for the classical Petersson-Weil metric on the Teichmüller space of Riemann surfaces of genus larger than one can be generalized:

Let $f: \mathcal{X} \rightarrow S$ be a family of Kähler manifolds with negative first Chern class over a (reduced) space $S$. According to the Calabi-Yau theorem there exist unique Kähler metrics $\omega_{\mathcal{X}_{s}}, s \in S$ with some fixed $k<0$, say $k=-1$. The relative volume forms $g(s)$ consitute: constitute a hermitian metric on the relative anti-canonical bundle $\mathcal{K}_{\mathcal{X} / S}^{-1}$. The $(2 \pi / k)$-fold of the Chern form now is a real, locally $\partial \bar{\partial}$-exact $(1,1)$-form $\omega_{\mathcal{X}}$ of class $C^{\infty}$ on $\mathcal{X} .{ }^{5}$ The Kähler-Einstein condition implies that all restrictions $\omega_{\mathcal{X}} \mid \mathcal{X}_{s}$ equal $\omega_{\mathcal{X}_{s}}$.

Observe that this construction also works for universal families of KählerEinstein manifolds with positive curvature. (For existence theorems cf. [TI 1987] and [T-Y 1987]).

Although the form $\omega_{\mathcal{X}}$ is only positive definite, when restricted to fibers, it can serve to define horizontal lifts of tanget: tangent vectors of the base. Such horizontal lifts give now rise to distinguished representatives of the KodairaSpencer classes.

We have $\omega_{\mathcal{X}}=-(1 / k) \sqrt{-1} \partial \bar{\partial} \log g(z, s)$.

The horizontal lift of $\partial / \partial s$ is just

$$
a^{\alpha}(z) \partial / \partial z^{\alpha}+\partial / \partial s
$$

where

$$
a_{\bar{\beta}}=\frac{1}{k} \frac{\partial^{2} \log g}{\partial z^{\bar{\beta}} \partial s} .
$$

(We use covariant derivatives as well as raising and lowering of indices with respect to our metrics on the fibers, e.g. $a_{\bar{\beta}}=g_{\alpha \bar{\beta}} a^{\alpha}$ ). Now (4) is automatic and (3) follows from a direct computation.

We remark that horizontal lifts in fact coincide with canonical lifts of tangent vectors in the sense of Siu [SI 1986].

Let $n$ be the dimension of the fibers of our family. The fiber-integral of the $(n+1)$-fold power of the Chern form of the relative anti-canonical bundle equipped with our metric $g$ is a real $(1,1)$-form.

5 The strategy, how to get differentiability for singular spaces $S$ is as follows: Given a holomorphic family of compact complex manifolds $\mathcal{X}_{s}$, fix the underlying differentiable structure and embed $S$ locally in some smooth space. The corresponding family of holomorphic structures can be extended to a differentiable family of almost complex structures. Now all relevant differential operators on the fibers can be extended differentiably to the ambient space. For the differentiability of the Kähler-Einstein metrics with respect to the parameter refer to the next part. 
Theorem. The generalized Petersson-Weil form on the base of a universal family of Kähler-Einstein manifolds of Ricci curvature $k \neq 0$ can be represented as a fiber-integral:

$$
\omega_{P W}=\alpha_{n} \int_{\mathcal{X} / S} c_{1}^{n+1}(\mathcal{X} / S, g)
$$

where

$$
\alpha_{n}=-\frac{2 \pi^{n+1}}{k^{n}(n+1) !}
$$

In particular $\omega_{P W}$ possesses locally (also around singular points) a $\partial \bar{\partial}-$ potential of class $C^{\infty}$.

For $n=1(9.5)$ is exactly Wolpert's formula.

The existence of a differentiable Kähler potential of $\omega_{P W}$ follows from the integral formula by means of a result of Varouchas [VA 1989] since the Chern form has this property.

We indicate, how to prove (9.5): Both sides of the equation define hermitian forms, hence one has to compare the induced (semi-)norms of any tangent vector $\partial / \partial s \in T_{s_{0}}(S)$. Therefore it is sufficient to restrict the map $f$ to the subspace of the first infinitesimal neighborhood of $s_{0} \in S$ which is defined by $\partial / \partial s$ and its preimage under the map $f$ whose reduction equals the fiber $X$. We can assume that $S$ is already of this kind.

Now the right hand side of (9.5) equals

$$
\frac{(-\sqrt{-1})^{n+1}}{(2 k)^{n}}\left(\int_{X} \phi d z^{1} \wedge d z^{\overline{1}} \wedge \ldots \wedge d z^{\bar{n}}\right) d s \wedge d \bar{s}
$$

where $\phi$ is the determinant of the complex Hessian of $\log g$ with respect to the coordinates $\left(z^{1}, \ldots, z^{n}, s\right)$. The Kähler-Einstein condition

$$
\frac{\partial^{2} \log g}{\partial z^{\alpha} \partial z^{\bar{\beta}}}=-k g_{\alpha \bar{\beta}}
$$

yields

$$
\phi=(-k)^{n}\left(\frac{\partial^{2} \log g}{\partial s \partial \bar{s}}+k \bar{a}_{\alpha} a_{\bar{\beta}} g^{\alpha \bar{\beta}}\right)
$$

The partial derivative with respect to $s$ and $\bar{s}$ can be eliminated using:

$$
\frac{1}{(-k)^{n} g}\left(\phi+\frac{1}{k} \square \phi\right)=A_{\bar{\beta}}^{\alpha} \bar{A}_{\gamma}^{\bar{\delta}} g^{\alpha \bar{\delta}} g_{\gamma \bar{\beta}},
$$

from which (9.4) follows finally. 


\subsection{A Positive Line Bundle on the Moduli Space of Canonically Polarized Manifolds and the Petersson-Weil Metric}

Determinant Line Bundles. Let $f: \mathcal{X} \rightarrow S$ be a universal family of canonically polarized manifolds. As for the construction of a positive line bundle on $S$, one can certainly not expect an entire analogy to the one-dimsional situation. The determinants of the sheaves $f_{*}\left(\mathcal{K}_{\mathcal{X} / S}^{\otimes k}\right)$ may not be positive and proportional for $k \in \mathbb{N}$. The problem is to find a suitable substitute for such a bundle. Donaldson [DO 1987] uses in a somewhat different situation virtual vector bundles - an approach which works also in a more general way.

Let $f: \mathcal{X} \rightarrow S$ be for the moment any family of compact complex manifolds and $\mathcal{F}$ a coherent sheaf on $\mathcal{X}$. Then the theory of Knudsen-Mumford [K-M 1976] associates to the direct image $\mathbf{R}{ }^{\bullet} f_{*} \mathcal{F}$ an invertible $\mathcal{O}_{S}$-module called the determinant of $\mathbf{R}^{\bullet} f_{*} \mathcal{F}$. ${ }^{6}$ One uses the notation $\lambda(\mathcal{F}):=\left(\operatorname{det} \mathbf{R}^{\bullet} f_{*} \mathcal{F}\right)^{-1}$. Let $\mathcal{F}$ be locally free and let the corresponding vector bundle be equipped with a hermitian metric $h_{\mathcal{F}}$. Assume that the fibers $\mathcal{X}_{s}$ carry a differentiable family of Kähler metrics. Then there exists on the determinant bundle a distinguished hermitian metric $k_{\mathcal{F}}$, the Quillen metric.

Chern Forms of Determinant Bundles. The theorem of Bismut, Gillet and Soulé [B-G-S 1987] states that the Chern form of the determinant bundle can be evaluated as a fiber integral:

$$
c_{1}\left(\lambda\left(\mathcal{F}, k_{\mathcal{F}}\right)\right)=-\left[\int_{\mathcal{X} / S} \operatorname{td}(\mathcal{X} / S, g) \operatorname{ch}\left(\mathcal{F}, h_{\mathcal{F}}\right)\right]_{2}
$$

where []$_{2}$ denotes the component of degree $2, \operatorname{ch}\left(\mathcal{F}, h_{\mathcal{F}}\right)$ and $\operatorname{td}(\mathcal{X} / S, g)$ the Chern and Todd character forms resp.

The formula should be considered a generalization of the Grothendieck Riemann-Roch theorem to Chern forms rather than classes.

On may remark that by checking details of the proof and using the methods of [A-G 1962], [F-K 1972] and [S 1972] concerning relative analytic spaces, the theorem of Bismut, Gillet and Soule can be extended to the case, where $S$ is singular.

We call an expression of the form $\mathcal{E}=\mathcal{F}-\mathcal{G}$, where $\mathcal{F}, \mathcal{G}$ are holomorphic vector bundles on $\mathcal{X}$, a virtual vector bundle and define $\lambda(\mathcal{E}):=\lambda(\mathcal{F}) \otimes \lambda(\mathcal{G})^{-1}$. Its Quillen metric is $k_{\mathcal{E}}:=k_{\mathcal{F}} \cdot k_{\mathcal{G}}^{-1}$. The construction of both the determinant bundle and the Quillen metric is compatible with base change. If $h_{\mathcal{F}}$ and $h_{\mathcal{G}}$ are hermitian metrics, then the Chern character form of the virtual bundle is defined by $\operatorname{ch}\left(\mathcal{E}, h_{\mathcal{E}}\right):=\operatorname{ch}\left(\mathcal{F}, h_{\mathcal{F}}\right)-\operatorname{ch}\left(\mathcal{G}, h_{\mathcal{G}}\right)$. Since both sides of (9.6) are additive, the formula holds also for virtual bundles.

An essential device consists in evaluating the Chern character form for virtual bundles of degree zero, like

\footnotetext{
6 The direct image of $\mathcal{F}$ equals in the derived category locally with respect to $S$ a bounded complex of free sheaves of finite type on $S$, for which the determinant sheaf is defined in the obvious way as product of determiniants: determiniants with alternating exponents \pm 1 .
} 


$$
\begin{aligned}
& \mathcal{E}=\left(\mathcal{L}-\mathcal{L}^{-1}\right)^{\otimes n} \otimes\left(\mathcal{K}_{\mathcal{X} / S}-\mathcal{K}_{\mathcal{X} / S}^{-1}\right) \\
& \mathcal{F}=\mathcal{L} \otimes\left(\mathcal{L}-\mathcal{L}^{-1}\right)^{\otimes n}
\end{aligned}
$$

where $\mathcal{L}$ is a hermitian line bundle on $\mathcal{X}$.

For these, the lowest terms of the Chern character forms are in degree $(n+1, n+1)$ and $(n, n)$ resp.

Proposition. Let $\mathcal{E}$ and $\mathcal{F}$ be the above virtual line bundles. Then

$$
c_{1}\left(\lambda\left(\mathcal{E}, k_{\mathcal{E}}\right)\right)=-2^{n+1} \int_{\mathcal{X} / S} c_{1}(\mathcal{L}, h)^{n} c_{1}(\mathcal{X} / S, g)
$$

and

$$
c_{1}\left(\lambda\left(\mathcal{F}, k_{\mathcal{F}}\right)\right)=2^{n-1} \int_{\mathcal{X} / S} c_{1}(\mathcal{L}, h)^{n} c_{1}(\mathcal{X} / S, g)-2^{n} \int_{\mathcal{X} / S} c_{1}(\mathcal{L}, h)^{n+1}
$$

Construction of the Line Bundle. For $\mathcal{L}=\mathcal{K}_{\mathcal{X} / S}$ our fiber-integral formula (9.5) and formula (9.7) yield:

Theorem. Let $\mathcal{X} \rightarrow S, \omega_{\mathcal{X} / S}$ be a universal family of Kähler-Einstein manifolds of non-zero curvature $k$. Then there exists a natural hermitian line bundle on $S$, whose Chern form is up to a numerical constant the Petersson-Weil form. Namely

$$
\omega_{P W}=\beta_{n} c_{1}\left(\lambda\left(\left(\mathcal{K}_{\mathcal{X} / S}-\mathcal{K}_{\mathcal{X} / S}^{-1}\right)^{n+1}\right), \tilde{k}\right)
$$

where

$$
\beta_{n}=\frac{(-\pi)^{n}}{2^{n} k^{n}(n+1) !},
$$

(the Quillen metric on the respective line bundle being denoted by $\tilde{k}$ ).

This line bundle descends to the moduli space of canonically polarized varieties. Its hermitian metric also descends (to a continous: continuous metric).

As mentioned before, the moduli space is locally the quotient of the base spaces of versal deformations by finite groups of automorphisms - it may be called a $V$-space in an analogous way to a $\mathrm{V}$-manifold. The generalized Petersson-Weil metric on $S$ satisfies the strongest possible condition on differentiability on $S$ - its $\partial \bar{\partial}$-potential on the quotient may just be continous: continuous though. 


\section{Moduli Spaces of Extremal Kähler Manifolds}

\subsection{Construction of the Moduli Space}

Extremal Kähler Metrics. Let $\left(X, \lambda_{X}\right)$ be a polarized Kähler manifold. According to Calabi [CA 1979, 1985] one considers on the space $U$ of all Kähler forms $\omega$ on $X$ which represent the polarization, the following functional $\Phi$ defined by

$$
\Phi(\omega):=\int_{X} R^{2}(\omega) \omega^{n}, \quad \omega \in U, \quad n=\operatorname{dim} X
$$

where $R$ denotes the scalar curvature $R=g^{\alpha \bar{\beta}} R_{\alpha \bar{\beta}}$ of $\omega$.

Definition. A critical point of the functional $\Phi$ is called an extremal Kähler form on $X$.

The Euler-Lagrange equation of $\phi$ is (cf. [CA 1982])

$$
R_{; \bar{\beta} \bar{\delta}}=0 \text {. }
$$

Remark. The Euler-Lagrange equation just means that the scalar curvature as a differentiable function gives rise to a holmorphic: holomorphic vector field, since (10.2) is equivalent to

$$
\bar{\partial}\left(g^{\alpha \bar{\beta}} R_{; \bar{\beta}} \frac{\partial}{\partial z^{\alpha}}\right)=0
$$

or in the notation of [CA 1982]

$$
\sqrt{-1} \bar{\partial} \uparrow_{g} \bar{\partial} R=0 .
$$

One can see that any Kähler manifold of constant scalar curvature, in particular any Kähler-Einstein manifold, is extremal.

Let $J: \operatorname{Aut}^{0}(X) \rightarrow A l b(X)$ be the Jacobi map of an arbitrary polarized manifold. Then the Lie algebra $a(X)$ of its kernel consists of those holomorphic vector fields which are orthogonal to holomorphic one-forms with respect to the natural pairing and thus coincides with the set of holomorhic; holomorphic vector fields originating from covariant derivatives of complex-valued differentiable functions by "pulling up indices". Note that ker $J$ is a linear algebraic group by a theorem of Fujiki [FU 1978].

Thus the compactness of $\mathrm{Aut}^{0}(X)$ is equivalent to

$$
a(X)=0
$$

We call this condition $(A)$.

Under this condition, any versal deformation is universal (cf. section 8).

By a theorem of Matsushima [MA 1957], Lichnerowitz [LI 1959] and Calabi [CA 1979] on an extremal Kähler manifold the Lie algebra of holomorphic vector 
fields is the direct sum of the ideal $\mathrm{a}(X)$ and the algebra of parallel holomorphic vector fields.

In particular, on an extremal Kähler manifold $\left(X, \omega_{X}\right)$ with compact $\operatorname{Aut}^{0}(X)$ any biholomorphic map in this group is an isometry. Moreover, any extremal Kähler manifold with $\mathrm{a}(X)=0$ ) is obviously of constant scalar curvature; although we will allways: always have to assume $(A)$ from now on, we will keep the notion of extremal manifolds. (If we waive this condition, holomorphic families of extremal Kähler manifolds are hard to define).

Deformations of Extremal Manifolds. In our situation, all versal deformations of a manifold $X$ with condition $(A)$ are universal (cf. section 8 ), we may expect that the combination of extremality and compactness of $\operatorname{Aut}^{0}(X)$ yields the assumptions of our criterion for the existence of a moduli space.

What is the correct definition of a family of extremal Kähler manifolds? In any case, it should induce a polarized family.

Definition. A family $\underline{\mathcal{X}}_{e}$ of extremal Kähler manifolds is a family of compact manifolds $f: \mathcal{X} \rightarrow S$ together with a $C^{\infty}$-family of extremal Kähler metrics $\tilde{\omega}=\left\{\omega_{s}\right\}$ on the fibers $\mathcal{X}_{s}, s \in S$ which represent a polarization.

This becomes a reasonable definition from the analytic viewpoint because of the following

Theorem. Let $\left(f: \mathcal{X} \rightarrow S, \lambda_{\mathcal{X} / S}\right)$ be a family of polarized Kähler manifolds with condition $(A)$ and $\left(X, \lambda_{X}\right)$ the fiber of a point $s_{0} \in S$. Let $\omega_{X}$ be an extremal Kähler form which represents the polarization. Then $\omega_{X}$ possesses an extension to a $C^{\infty}$-family of extremal Kähler metrics on the neighboring fibers which represents the polarization, uniquely.

As usual, the notion of a "holomorphic familiy: family" generates the notion of a deformation:

Corollary. Any compact extremal Kähler manifold $X$ with compact $\operatorname{Aut}^{0}(X)$ posseses a universal deformation.

Such universal deformations are just universal deformations of the underlying polarized families with extremal metrics extended according to the theorem.

We indicate the proof of the theorem: As observed in section 8, we can extend $\omega_{X}$ to a loacally $\partial \bar{\partial}$-exact $(1,1)$-form $\tilde{\omega}_{\mathcal{X}}$ of class $C^{\infty}$ (even if $S$ is singular). Our approach is to set

$$
\omega_{\mathcal{X}}=\tilde{\omega}_{\mathcal{X}}+\sqrt{-1} \partial \bar{\partial} \Phi,
$$

where $\Phi$ is a real-valued function, uniquely determined by $\omega_{\mathcal{X}}$ up to a function on $S$ which can be normalized. Using a differentiable trivialization of the underlying holomorphic family, one considers $\Phi$ as a map from the base with values in a Sobolev space $H^{k}(X)$ of normalized differentiable functions on the fiber $X$. (Constant functions 
are zero). Any $s \in S$ and function $\psi \in H^{k}(X)$ close to $s_{0}$ and zero resp. determine a Kähler form on the respective fiber. Denote by $R(\psi, s)$ the scalar curvature of the Kähler form corresponding to $\psi$ and $s$. Then $R(\psi, s)$ can be considered as a map with values in $H^{k-4}(X)$ up to some error term which is due to the fact that the integral of the scalar curvature over a fiber is no longer a constant, if we use a trivialization and a fixed volume form. We want to solve the equation $R(s, \psi(s))=$ const. by means of the generalized implicit function theorem. The partial derivative $L: H^{k}(X) \rightarrow H^{k-4}(X)$ of this function with respect to the second derivative at $\left(s_{0}, 0\right)$ is

$$
L(\phi)=g^{\alpha \bar{\beta}} g^{\gamma \bar{\delta}}\left(\phi_{; \alpha \bar{\beta} \gamma \bar{\delta}}+R_{\bar{\beta} \gamma} \phi_{; \alpha \bar{\delta}}\right),
$$

in terms of the metric and Ricci tensor on the central fiber $X$. A direct computation, based upon $R=$ const. on $X$, shows that

$$
L(\phi)=g^{\alpha \bar{\beta}} g^{\gamma \bar{\delta}} \phi_{; \bar{\beta} \bar{\delta} \alpha \gamma} .
$$

In this setting, the assumption $a(X)=0$ is applicable; one can see that the elliptic operator $L$ has a bounded inverse. The claim follows from classical regularity theorems.

Remark. Although the extremal metrics on the neighboring fibers are uniquely determined, uniqueness does in general not hold for the inducing locally $\partial \bar{\partial}-$ exact $(1,1)$-form $\omega_{\mathcal{X}}$ unless the first Betti number of the fibers vanishes.

Another consequence of the theorem concerns isomorphisms of families of extremal manifolds:

Let $\underline{\mathcal{X}}_{e}=(\mathcal{X} \rightarrow S, \tilde{\omega})$ and $\underline{\mathcal{Y}}_{e}=\left(\mathcal{Y} \rightarrow S, \tilde{\omega}^{\prime}\right)$ be families of extremal Kähler manifolds with $(A)$, and $\underline{\mathcal{X}}, \underline{\mathcal{Y}}$ the underlying families of polarized manifolds.

Proposition. The isomorphism functor of extremal families is representable by a a: a complex space $I_{e}=\operatorname{Isom}_{S}\left(\underline{\mathcal{X}}_{e}, \underline{\mathcal{Y}}_{e}\right) \rightarrow S$ such that the natural map from $I_{e}$ to the classifying space $I$ of isomorphisms between $\underline{\mathcal{X}}$ and $\underline{\mathcal{Y}}$ is locally biholomorphic. The map $I_{e} \rightarrow S$ is proper.

The first claim follows from the theorem. Since we only know that extremal metrics are $\operatorname{Aut}^{0}(X)$-invariant (under condition $(\mathrm{A})$ ), and no general uniqueness or existence theorem is known, one cannot say more. The classifying space of isomorphisms carries the topology of compact convergence. As isomorphisms of extremal Kähler manifolds are isometries, the properness follows from classical theorems mentioned in the beginning of our discussion.

All of the preceeding: preceding considerations can be realized for extremal Hodge manifolds, i.e. for Hodge manifolds $(X, L)$ equipped with an extremal Kähler form which represents the Chern class of $L$.

We are now able to apply our criterion:

Theorem. There exist the moduli spaces $\mathcal{M}_{e}$ and $\mathcal{M}_{H, e}$ resp. of extremal Kähler and extremal Hodge manifolds resp. for which the identity components of the automorphism groups are compact. 
Remark. The above theorem includes also the existence of the moduli space of Kähler-Einstein manifolds of positive curvature with compact automorphism groups.

\subsection{The Generalized Petersson-Weil Metric}

It was impossible to generalize directly the Petersson-Weil metric to the case of extremal Kähler manifolds. The approach to use harmonic representatives of Kodaira-Spencer classes failed. Any proof of the Kähler property had to depend upon a close relationship with certain symmetric tensors - like the infinitesimal deformations of the metric tensor of a Kähler-Einstein metric. The answer is based upon a formula of Berger and Ebin [B-E 1969] for the derivative of the scalar curvature with respect to some parameter an: and a decomposition theorem for symmetric tensors in the same article. The proper distinguished representatives satisfy a forth order elliptic equation, and from the definition, it is possible to verify the Kähler condition by a lengthy computation - at least when the base is smooth. Here we shall present a fiber integral formula and an approach to the Petersson-Weil metric which proved to be very natural - a posteriori.

Infinitesimal Deformations of Polarized Manifolds. For a family $f: \mathcal{X} \rightarrow S$ of complex manifolds (always assume (A)) and a polarization $\lambda_{X}$ of a fiber $X$, the obstructions against its extension consisted in a holomorphic section of $\mathbf{R}^{2} f_{*} \mathcal{O}_{\mathcal{X}}$ (cf. section 8). Infinitesimally the assignment of a tangent vector of $S$ to its obstruction is (via the Kodaira-Spencer map) just the map $\cup_{\lambda_{X}}: H^{1}\left(X, \Theta_{X}\right) \rightarrow H^{2}\left(X, \mathcal{O}_{X}\right)$, induced by the cup-product. Its kernel $H^{1}\left(X, \Theta_{X}\right)_{\lambda_{X}}$ is the space of infinitesimal deformations of $\left(X, \lambda_{X}\right)$. With respect to the canonical isomorphism $H^{1}\left(X, \Theta_{X}\right) \stackrel{\sim}{\rightarrow} \operatorname{Ext}\left(X, \mathcal{O}_{X}\right)$ the given polarization $\lambda_{X}$ corresponds to the isomorphism class of an extension

$$
0 \rightarrow \mathcal{O}_{X} \rightarrow \Sigma_{X} \rightarrow \Theta_{X} \rightarrow 0
$$

If the polarization is the Chern class of an element $L \in \operatorname{Pic}(X)$ the sequence (10.3) is just the Atiyah-sequence, and $H^{1}\left(X, \Sigma_{X}\right)$ is the space of infinitesimal deformations of the Hodge manifold ${ }^{7}(X, L)$, and $H^{0}\left(X, \Sigma_{X}\right)$ is the space of infinitesimal automorphisms of $(X, L)$.

The induced cohomology sequence may give some more insight. We assume $a(X)=0$, then the following is exact.

$$
0 \rightarrow H^{0}\left(X, \Theta_{X}\right) \stackrel{\delta_{0}}{\rightarrow} H^{1}\left(X, \mathcal{O}_{X}\right) \rightarrow H^{1}\left(X, \Sigma_{X}\right) \rightarrow H^{1}\left(X, \Theta_{X}\right) \stackrel{\delta_{1}}{\rightarrow} H^{2}\left(X, \mathcal{O}_{X}\right) \cdots
$$

The edge-homomorphisms $\delta_{0}$ and $\delta_{1}$ are just given by the cup product with the polarization. The map $\delta_{0}$ is induced by the infinitesimal action of $\operatorname{Aut}^{0}(X)$ on $\operatorname{Pic}(X)$.

7 In the general case it corresponds to infinitesimal deformations of the manifold $X$ equipped with a refined Kähler class. 
The Generalized Petersson-Weil Metric. Let $(\mathcal{X} \rightarrow S, \mathcal{L}, \tilde{\omega})$ be a family of polarized, extremal Hodge manifolds $-\mathcal{L}$ is a section of the relative Picard group and $\tilde{\omega}$ a family of extremal Kähler metrics. Let $\mathcal{L}$ be represented by an actual line bundle denoted by the same letter. It is desirable to construct a hermitian metric $h$ on $\mathcal{L}$ such that $\omega_{\mathcal{X}}:=2 \pi c_{1}(\mathcal{L}, h)$ induces on all fibers the given extremal metrics. Starting from an arbitrary metric on $\mathcal{L}$ the method described in section 10.1 works - and in a sense the ambiguity is taken from $h$ and $\omega_{\mathcal{X}}$. These are now unique up to a factor and summand resp. which depend only on the parameter $s \in S .{ }^{8}$

Now as in the Kähler-Einstein case (cf. section 9.1), we take horizontal lifts of tangent vectors $\partial / \partial s$ of the base of the family which give rise to certain representatives $\eta=B_{\bar{\beta}}^{\alpha} \partial / \partial z^{\alpha} d z^{\bar{\beta}}$ of the associated Kodaira-Spencer class on the fiber $X$. A computation like in the Kähler-Einstein case furnishes the following equations:

$$
\begin{gathered}
B_{\bar{\beta} \bar{\delta}}=B_{\bar{\delta} \bar{\beta}} \\
g^{\bar{\beta} \alpha} g^{\bar{\delta} \gamma} B_{\bar{\beta} \bar{\delta} ; \alpha \gamma}=0 \\
B_{\bar{\beta} \bar{\delta} ; \bar{\tau}}=B_{\bar{\beta} \bar{\tau} ; \bar{\delta}}
\end{gathered}
$$

Equation $(a)$ is the relationship of $\eta$ with the first derivative of the metric tensor, and (c) is the $\bar{\partial}$-closedness of $\eta$, or the infinitesimal integrability condition of the almost complex structure $B_{\bar{\beta}}^{\alpha}$.

The second formula $(b)$ can be recognized easily as the equation for the constancy of the scalar curvature (cf. [B-E 1969]), where all terms but one vanish on a Kähler manifold. It is related to decomposition theorems of spaces of symmetric tensors with respect to certain elliptic operators in the same article. (A more general construction related to an elliptic complex is due to Calabi [CA 1960]). It just says that $B_{\bar{\beta} \bar{\delta}}$ is orthogonal with respect to the $L^{2}$-inner product to the space of tensors of the form

$$
f_{;} \bar{\beta} \bar{\delta}
$$

where $f$ is a $C^{\infty}$-function. One can check in different ways that $(10.5)(a)-(c)$ define distinguished representatives of classes in $H^{1}\left(X, \Sigma_{X}\right)$. The image of $B_{\bar{\beta} \bar{\delta}}$ in $H^{1}\left(X, \Theta_{X}\right)$ is the class of $\eta$. Given a harmonic $(0,1)$-form $u_{\bar{\beta}} d z^{\bar{\beta}}$ on $X$, its image in $H^{1}\left(X, \Sigma_{X}\right)$ equals $u_{\bar{\beta} ; \bar{\delta}} d z^{\bar{\beta}} \wedge d z^{\bar{\delta}}$ Thus, one can identify the kernel of $\delta^{1}$, i.e. the space of infinitesimal deformations of the underlying polarized manifold with the space of tensors $A_{\bar{\beta} \bar{\delta}}$ which satisfy the additional condition

$(10.5)(d) \quad \int_{X} A_{\bar{\beta} \bar{\delta}} v_{\alpha ; \gamma} g^{\bar{\beta} \alpha} g^{\bar{\delta} \gamma} g d v=0$, for all holomorphic 1-forms $v_{\alpha} d z^{\alpha}$.

8 The analogous construction works for manifolds with refined Kähler classes. So it can also be applied in the Kähler case. 
Definition. Let a universal family of extremal, polarized Kähler or Hodge manifolds be given. Associate to any Kodaira-Spencer class the distinguished representative satisfying $(10.5)(a)-(d)$ or (a)-(c) resp. Then the generalized Petersson-Weil metric on the base of such a family is defined by the $L^{2}$-inner product (cf. (9.2)) of these tensors. We denote the corresponding differential form on $S$ by $\hat{\omega}_{P W}$.

Remark. There is a natural map from the moduli space of (extremal) polarized Hodge manifolds to the moduli space of polarized Kähler manifolds. This map is also defined on the level of universal families. The fibers are smooth related to the quotients of the form $H^{1}\left(X, \mathcal{O}_{X}\right) / H^{0}\left(X, \Theta_{X}\right)$. One can read of the formula of the Petersson-Weil metric that it is flat, when restricted to the fibers.

A Fiber-Integral Formula. Our general situation can be characterized as follows: We are given a family $f: \mathcal{X} \rightarrow S$ of compact complex manifolds over a complex space $S$ (which need not be even reduced at this place), and a $(1,1)$-form $\omega_{\mathcal{X}}$ on $\mathcal{X}$ which has locally a real $\partial \bar{\partial}$-potential of class $C^{\infty}$ and induces on all fibers Kähler metrics. Then horizontal lifts of tangent vectors of the base are welldefined, (even if $\omega_{\mathcal{X}}$ is only fixed up to some form $f^{*}\left(\omega_{S}\right)$, where $\omega_{S}$ is a locally $\partial \bar{\partial}$-exact $(1,1)$-form on $S$ ). Such a differential form may be called admissible.

As discussed above the horizontal lift of tangent vectors furnishes representatives of the Kodaira-Spencer classes related to symmetric 2-tensors, and the $L^{2}$-inner product of such constitutes a hermitian form on the tangent space of $S$ at the respective point. The form is positive definite, provided the family is versal. Denote by $\Omega_{S}$ the corresponding $(1,1)$-form on $S .^{9}$

An admissible form $\omega_{\mathcal{X}}$ induces a hermitian metric $g$ on the relative anticanonical bundle.

Theorem. Let $\mathcal{X} \rightarrow S, \omega_{\mathcal{X}}$ be as above. Then

$$
\Omega_{S}=-\int_{\mathcal{X} / S}\left(2 \pi c_{1}(\mathcal{X} / S, g) \frac{\omega^{n}}{n !}-R \frac{\omega^{n+1}}{(n+1) !}\right)
$$

(Here $R$ is the differentiable function, whose restriction to a fiber is the respective scalar curvature of $\omega_{\mathcal{X}} \mid \mathcal{X}_{s}$ ). $C^{\infty}$

In particular, the real form $\Omega_{S}$ possesses locally a $\partial \bar{\partial}$-potential of class

The proof depends on the methods used in the Kähler-Einstein case (cf. section 9.1$)$.

Let $(\mathcal{X} \rightarrow S, \mathcal{L}, \tilde{\omega})$ be a family of extremal polarized Hodge manifolds with a hermitian metric $h$ and global $(1,1)$-form $\omega_{\mathcal{X}}=2 \pi c_{1}(\mathcal{L}, h)$ arranged like in

9 Although the dimension of the tangent space need not be constant, differentiability with respect to the parameter is not a problem (cf. footnote 5). 
the preceding section. The general theorem above now yields a fiber-integral formula for the Petersson-Weil form:

Corollary. The Petersson-Weil form on the base of a universal family of extremal Hodge manifolds equals

$$
\hat{\omega}_{P W}=-(2 \pi)^{n+1}\left(\int_{\mathcal{X} / S} c_{1}(\mathcal{X} / S, g) \frac{c_{1}(\mathcal{L}, h)^{n}}{n !}-R \int_{\mathcal{X} / S} \frac{c_{1}(\mathcal{L}, h)^{n+1}}{(n+1) !}\right)
$$

in particular, $\hat{\omega}_{P W}$ is a Kähler form which possesses locally a Kähler potential. ${ }^{10}$

The value of the scalar curvature is in terms of the cohomology classes

$$
R=c_{1}\left(\mathcal{X}_{s}\right) \cdot c_{1}\left(\mathcal{L}_{s}\right)^{n-1} / c_{1}^{n}(\mathcal{L})^{n}
$$

which does not depend on the parameter $s$.

The contribution from the second term in (10.7) can be cancelled: canceled in the following way: If $\int \omega_{\mathcal{X}}^{n+1}=\eta$, replace $\omega_{\mathcal{X}}$ by $\omega_{\mathcal{X}}-\alpha^{-1} f^{*} \eta$, where $\alpha=$ $(n+1) \int_{\mathcal{X} / S} \omega_{\mathcal{X}}^{n}$ is up to a factor just the (constant) volume of the fibers. This does not affect horizontal lifts neither the metrics on the fibers.: This affects neither the horizontal lift nor a metric on the fibers. This adjustment can be raised locally with respect to $S$ to the level of hermitian metrics on $\mathcal{L}$, since $\eta$ has a $\partial \bar{\partial}$-potential. One may call such admissible forms and hermitian metrics normalized and make up a deformation theory in this way. However, the hermitian metric on $\mathcal{L}$ is only unique up to a constant, a fact which caused a problem, when putting a positive bundle on the moduli space.

Corollary. The Petersson-Weil form on the base of a universal family of extremal Kähler manifolds possesses locally a Kähler potential of class $C^{\infty}$.

If the first Betti number of the fiber vanishes, or more generally, if $b_{1}$ equals the dimension of $\operatorname{Aut}(X)$, the corollary follows immediately from (10.7). Otherwise: Otherwise, one has to use the remark in section 10.2. For details cf. [F-S 1988b]).

\subsection{Positive Line Bundles}

We indicate how to construct a positve: positive line bundle on the moduli spaces of extremal Hodge manifolds and extremal Kähler manifolds (with integer-valued or rational) polarization, whose curvature is up to some numerical constant the Petersson-Weil form $\hat{\omega}_{P W}$ or $\omega_{P W}$.

Local Construction. Let $(f: \mathcal{X} \rightarrow S, \mathcal{L}, \tilde{\omega})$ be a family of extremal Hodge manifolds. The idea is to combine the formulas (9.7) and (9.8) for the Chern forms of the determinat: determinant bundles arising from the virtual bundles

${ }^{10}$ Again there is an analogous theorem for families of extremal Kähler manifolds with refined Kähler classes. 
$\mathcal{E}=\left(\mathcal{L}-\mathcal{L}^{-1}\right)^{\otimes n} \otimes\left(\mathcal{K}_{\mathcal{X} / S}-\mathcal{K}_{\mathcal{X} / S}^{-1}\right)$ and $\mathcal{F}=\mathcal{L} \otimes\left(\mathcal{L}-\mathcal{L}^{-1}\right)^{\otimes n}$ resp. with the fiber-integral expression (10.7) for $\hat{\omega}_{P W}$. This can be realized by a suitable linear combination of these bundles:

Denote by $a=c_{1}\left(\mathcal{X}_{s}\right) \cdot c_{1}\left(\mathcal{L}_{s}\right)^{n-1}$ and $b=c_{1}\left(\mathcal{L}_{s}\right)^{n}$ natural numbers (not depending on $s$ ) with $R=n a / b$ and set $\mathcal{G}=\mathcal{E}^{\oplus(n a-2(n+1) b)} \oplus \mathcal{F}^{\oplus 4 n a}$.

Theorem. The Petersson-Weil form on the base of a universal deformation of extremal Hodge manifolds equals up to a numerical constant the Chern form of the determinant bundle of a virtual vector bundle on the total space, equipped with the Quillen metric:

Namely

$$
\hat{\omega}_{P W}=\frac{-(\pi / 2)^{n+1}}{n !} c_{1}\left(\lambda\left(\mathcal{G}, k_{\mathcal{G}}\right)\right)
$$

For any universal deformation of an extremal Hodge manifold $\left(X, L, \omega_{X}\right)$ the action of the automorphism group of the fiber on the base $\left(S, s_{0}\right)$ descends to the determinant bundle $\lambda(\mathcal{G}, k)$ by functoriality of the construction. Denote by $G \subset \operatorname{Aut}\left(S, s_{0}\right)$ the associated finite group. Then $G$ acts trivially on some power $\lambda(\mathcal{G}, k)^{m}$ (e.g. take as $m$ the order of $\left.G\right)$. In such a situation the power of determinant bundle descends to a determinant bundle on $S / G$ and the Quillen metric descends to a continuous metric on the push-down. One may call this a hermitian line bundle with respect to the $\mathrm{V}$-structure or $\mathrm{V}$-hermitian line bundle.

Global Construction on the Moduli Space of Extremal Hodge Manifolds. First, we can decompose $\mathcal{M}_{H, e}$ into a disjoint union (of open and closed) subspaces $\mathcal{M}_{H, e}^{P}$ corresponding to isomorphism classes of $\left(X, L, \omega_{X}\right)$, where $P$ is the Hilbert polynomial of $L$. We consider such a component. We fix a uniform exponent such that all powers of the line bundles $L$ in question are very ample. Then one can show that such a $\mathcal{M}_{H, e}^{P}$ is essentially a quotient of an open subset $U$ in the corresponding Hilbert scheme. The main point is that one can construct a global family of extremal Hodge manifolds with a global G-invariant hermitian metric on the line bundle. Now the method explained in 10.1 yields globally a hermitian metric, whose Chern form is admissible for the family of extremal manifolds, i.e. its restriction to the fibers yields the extremal metrics. The construction of the determinant bundle as well as (10.8) can be globalized: A power of the determinant bundle, equipped with the Quillen metric exists globally on $\mathcal{M}_{e}^{P}$ and yields the Petersson-Weil metric. This is the content of the following

Theorem. The moduli space $\mathcal{M}_{H, \text { e }}$ of extremal polarized Hodge manifolds possesses a V-hermitian line bundle whose Chern form equals up to a numerical constant the Petersson-Weil form $\hat{\omega}_{P W}$. 
Denote by $\mathcal{M}_{e}^{\prime} \subset \mathcal{M}_{e}$ the space of extremal Kähler manifolds with integervalued polarization and by $p: \mathcal{M}_{H, e} \rightarrow \mathcal{M}_{e}^{\prime}$ the natural projection which assigns to an extremal Hodge manifold the underlying polarized Kähler manifold. Then $p$ is proper, its fibers are essentially the quotients of the Picard groups by the automorphism groups of the corresponding manifolds. We have seen in the remark following (10.5) that $\hat{\omega}_{P W}$ is flat, when restricted to the fibers of $p$. In particular on respective connected components of the moduli spaces (which we denote by the same letter)

$$
\omega_{P W}=\text { const. } \int_{\mathcal{M}_{H, e} / \mathcal{M}_{e}^{\prime}} \hat{\omega}_{P W}^{m+1},
$$

where $m$ is the dimension of the fibers of $p$.

On the other hand, let $(\mathcal{G}, k)$ be the determinant bundle on $\mathcal{M}_{H, e}$ which induces $\hat{\omega}_{P W}$. Then like in section 9.2 we get

$$
c_{1}\left(\left(\mathcal{G}-\mathcal{G}^{-1}\right)^{\otimes m+1}\right)=2^{m+1} \int_{\mathcal{M}_{H, e} / \mathcal{M}_{e}^{\prime}} c_{1}(\mathcal{G}, k)^{m+1} .
$$

Thus: Thus,

Theorem. The moduli space of $\mathcal{M}_{e}^{\prime}$ of extremal Kähler manifolds with integervalued polarization carries a hermitian line bundle, whose Chern form is up to a constant the Petersson-Weil form.

Corollary. All compact complex subspaces of the moduli spaces $\mathcal{M}_{e}^{\prime}$ and $\mathcal{M}_{H, e}$ resp. including the moduli spaces of Kähler-Einstein manifolds are projective.

Note added in proof:

In the meantime A. M. Nadel has introduced multiplier ideal sheaves in order to prove the existence of Kähler-Einstein metrics. Examples include Del Pezzo surfaces and complete intersections of low degree, the blow-up: blowup of $\mathbb{P}_{4}$ along the intersection of two quartic hypersurfaces, and the cubic threefold along an elliptic curve.

E. Viehweg has proved the quasi-projectivity of the moduli scheme of polarized compact manifolds (with fixed Hilbert polynomial) and semi-ample canonical bundle.

\section{References}

Abikoff, W. [AB 1980] The real analytic theory of Teichmüller space, Lect. Notes in Math. 820, Berlin, Heidelberg, New York 1980

Ahlfors, L.V [AH 1938a] On quasiconformal mappings, Journal d'Analyse Math. 3, 359-364 (1938)

Ahlfors, L.V [AH 1938b] An extension of Schwarz's lemma, Transactions of the AMS 43, 359-364 (1938)

Ahlfors, L.V [AH 1953] On quasiconformal mappings, J. d'Analyse Math.3, 1-58 (1953)

Ahlfors, L.V [AH 1960] The complex analytic structure of the space of closed Riemann surfaces, in Analytic Functions, Princeton University Press (1960) 
Ahlfors, L.V [AH 1961a] Some remarks on Teichmüller's space of Riemann surfaces, Ann. of Math. 74, 171-191 (1961)

Ahlfors, L.V [AH 1961b] Curvature properties of Teichmüller's space, J. d'Analyse Math. 9, 161-176 (1961)

Ahlfors,L., Bers, L. [A-B 1960] Riemann mapping theorem for variable metrics, Annals of. Math. 72 385-404 (1960)

Andreotti, A., Grauert, H. [A-G 1962] Théorème de finitude pour la cohomologie des espace complexes, Bull. Soc. Math. France 90, 193-259 (1962)

Baily, W.L. [BA 1957] On the imbedding of V-manifolds in projective space, Amer. J. Math. 79, 403-430 (1957)

Baily, W.L. [BA 1962] On the theory of $\Theta$-functions, the moduli of abelian varieties and the moduli space of curves, Ann. Math. 75, 342-381 (1962)

Baily, W.L., Borel, A. [B-B 1966] Compactification of arithmetic quotients of bounded symmetric domains, Ann. Math 84, 442-528 (1966)

Berger, M., Ebin, D.G. [B-E 1969] Some decompositions on the spaces of symmetric tensors on a Riemannian manifold, J. Diff. Geom. 3, 379-392 (1969)

Bers, L. [BE 1960] Spaces of Riemann surfaces, Proc. Int. Cong. 1958, Cambridge 1960

Bers, L. [BE 1970] On boundaries of Teichmüller spaces and Kleinian groups I, Ann. Math. 91, 570-600 (1970)

Bers, L. [BE 1974] Spaces of degenerating Riemann surfaces, discontinous groups and Riemann surfaces, Princeton University Press, Princeton 1974

Bismut, J.M., Gillet, H., Soulé, Ch. [B-G-S 1987] Analytic torsion and holomorphic determinant bundles, I, II, III, Comm. Math. Phys. 115, 49-87, 79-126, 301-351 (1987)

Calabi, E. [CA 1960] On compact Riemann manifolds with constant curvature. I, AMS Proc. Symp. Pure Math. III, 155-180 (1960)

Calabi, E. [CA 1979] Extremal Kähler metrics. In: Yau, S.T. (ed.) Seminars on differential geometry, Princeton 1979,

Calabi, E. [CA 1985] Extremal Kähler metrics II. In: Cheval, I., Farkas, H.M. (eds.) Differential geometry and complex analysis, dedicated to E. Rauch, Springer 1985, pp. $259-290$

Campana, F., Schumacher, G. [C-S 1989] A geometric algebraicity property for moduli spaces of compact Kähler manifolds with $h^{2,0}=1$, to appear in Math. Z.

Donaldson, S.K. [DO 1987] Infinite determinants, stable bundles and curvature, Duke Math. J. 54, 231-247 (1987)

Earle, C. J., Kra,I., [E-K 1974] On holomorphic mappings between Teichmüller spaces, in Contributions to Analysis, (Ahlfors, L., Kra, I., Maskit, B., Nirenberg, L. edts.), New York, London, 1974

Fenchel, W., Nielsen, J. [F-N] J. Discontinous groups of non-Euklidean motions. unpublished manuscript

Fischer, A.E., Tromba A.J. [F-T 1984a] On a purely Riemannian proof of the structure and dimension of the unramified moduli space of a compact Riemann surface, Math. Ann 267, 311-345 (1984)

Fischer, A.E., Tromba A.J. [F-T 1984b] On the Weil-Petersson metric on Teichmüller space, Trans. AMS 284, 311-345 (1984)

Fischer, A.E., Tromba, A.J. [F-T 1987] A new proof that Teichmüller's space is a cell, Trans. AMS 303, 257-262 (1987)

Forster. O., Knorr, K. [F-K 1972] Relativ-analytische Räume und die Kohärenz von Bildgarben, Invent. math 16, 113-160 (1972)

Fricke, R., Klein, F. [F-K 1926] Vorlesungen über die Theorie der automorphen Funktionen, Leipzig, 1926

Fujiki, A. [FU 1978] On automorphism groups of compact Kähler manifolds, Invent. math 44, 226-258 (1978)

Fujiki, A. [FU 1981] A theorem on bimeromorphic maps of Kähler manifolds and its applications, Publ. RIMS Kyoto 17, 735-754 (1981)

Fujiki, A. [FU 1984] Coarse moduli spaces for polarized Kähler manifolds, Publ. RIMS, Kyoto 20, 977-1005 (1984) 
Fujiki, A., Schumacher, G. [F-S 1988a] The moduli space of Kähler structures on a real symplectic manifold, Publ. RIMS, Kyoto 24 141-168 (1988)

Fujiki, A., Schumacher, G. [F-S 1988b] The moduli space of extremal, compact Kähler manifolds and generalized Weil-Petersson metrics, preprint 1988, 157 p., to appear in Publ. RIMS, Kyoto

Gerritsen, L., Herrlich, F. [G-H 1988] The extended Schottky space, J. reine angew. Math. 389, 190-208 (1988)

Gerstenhaber, M., Rauch, H.E. [G-R 1954] On extremal quasi-conformal mappings, I, II, Proc. Nat. Acad. Sci, 40, 808-812, 991-994 (1954)

Grötzsch, H. [GR 1928] Über die Verzerrung bei schlichten nichtkonformen Abbildungen und über eine damit zusammenhängende Erweiterung des Picardschen Satzes, Leipz. Ber. 80 (1928)

Grothendieck, A. [GR 1961] Technique de construction en géométrie analytique, Sém. Cartan no. 7-17 (1960/61)

Harer, J. [HA 1983] The second homology group of the mapping class group of an orientable surface, Invent. math. 72, 221-231 (1983)

Harris, J., Mumford, D. [H-M 1982] On the Kodaira dimension of the moduli space of curves, Invent. math. 67, 23-86 (1982)

Harris, J. [HA 1984] On the Kodaira dimension of the moduli space of curves, II. The even-genus case, Invent. math. 75, 437-466 (1984)

Herrlich, F. [HE 1989] The extended Teichmüller space, to appear in Math. Z.

Jost, J., Yau, S.T. [J-Y 1987] On the rigidity of certain discrete groups and algebraic varieties, Math. Ann. 278, 481-496 (1987)

Jost, J. [JO 1989] Harmonic maps and curvature computations in Teichmüller theory, preprint, 1989

Keen, L. [KE 1971] On Fricke moduli, Ann. of Math. Stdies 66, 205-224 (1971)

Knudsen, F., Mumford, D. [K-M 1976] The projectivity of the moduli space of stable curves, I: Preliminaries on "det" and "div", Math. Scand. 39, 19-55 (1976)

Knudsen, F. [KN 1983a] The projectivity of the moduli space of stable curves, II: The stacks $M_{g, n}$, Math. Scand. 52, 161-199 (1983)

Knudsen, F. [KN 1983b] The projectivity of the moduli space of stable curves, III: The line bundles on $M_{g, n}$ and a proof of the projectivity of $\overline{M_{g, n}}$ in characteristic 0 , Math. Scand. 52, 200-212 (1983)

Koiso, N. [KO 1983] Einstein metrics and complex structure, Invent. math. 73, 71-106 (1983)

Kra, I. [KR 1989] Horocyclic coordinates for Riemann surfaces and moduli spaces, I: Teichmüller and Riemann spaces of Kleinian groups, Preprint (1989)

Lichnerowicz, A. [LI 1959] Isométrie et transformations analytique d'une variété Kählerienne compacte, Bull. Soc. Math. France 87, 427-437 (1959)

Liebermann, P. [LI 1978] Compactness of the Chow Scheme: application to automorphisms and deformations of Kähler manifolds, Sém Norguet. Lecture Notes in Mathematics 670. Berlin Heidelberg, New York: Springer 1978

Maskit, B. [MT 1977] Decomposition of certain Kleinian groups, Acta math. 130, 63-82 (1977)

Matsushima, Y. [M 1957] Sur la structure du groupe d'homomorphismes analytique d'une certaine variété Kählerienne, Nagoya Math. J. 11, 145-150 (1957)

Mazur, H. [MA 1976] The extension of the Weil-Petersson metric to the boundary of Teichmüller space. Duke Math. J. 43, 623-635 (1976)

Mumford D. [MU 1977] Stability of projective varieties, L'enseign. math. 23 39-11 (1977)

Petersson, H. [PE 1949] Über die Berechnung der Skalarprodukte ganzer Modulformen. Comment. math. helv 22, 168-199 (1949)

Popp, H. [PO 1977] Moduli theory and classification theory of algebraic varieties, Lecture notes in Math. 620, Berlin, Heidelberg, New York, 1977

Richberg, R. [RB 1968] Stetig, streng pseudokonvexe Funktionen, Math. Ann. 175, 257-286 (1968)

Reich, E. [RE 1985] On the variational principle of Gerstenhaber and Rauch, Ann. Acad. Sci. Fenn. Ser. A I Math. 10, 469-475 (1985) 
Riemann B. [RI 1857] Theorie der Abel'schen Functionen.. Borchardt's Journal für reine und angewandte Mathematik, Bd 54 (1857)

Royden, H.L. [RO 1971] Automorphisms and isometries of Teichmüller space, Advances in the theory of Riemann surfaces, Stony Brook, 1969, Annals of Math. Studies, 66 (1971)

Royden, H.L. [RO 1974a] Invariant metrics on Teichmüller space, in Contributions to Analysis, (Ahlfors, L., Kra, I., Maskit, B., Nirenberg, L. edts.) New York, London, 1974

Royden; H.L. [RO 1974b] Intrinsic metrics on Teichmüller space, Proc. Int. Cong. Math. 2, 217-221 (1974)

Saito, Kyoji [SAI 1988] Moduli space for Fuchsian groups, Alg. Analysis II, 735-787 (1988)

Sampson, J.H. [SA 1978] Some properties and applications of harmonic mappings, Ann. Sci. École Norm. Sup. 4, 211-228 (1978)

Schneider, M. [S 1972] Halbstetigkeitssätze für relativ analytische Räume, Invent. math. 16, 161-176 (1972)

Schoen, R., Yau, S.T. [S-Y 1978] On univalent harmonic maps between surfaces, Invent. math 44, 265-278 (1978)

Schumacher, G. [SCH 1983] Construction of the coarse moduli space of compact polarized Kähler manifolds with $c_{1}=0$, Math. Ann. 264, 81-90 (1983)

Schumacher, G. [SCH 1984] Moduli of polarized Kähler manifolds, Math. Ann. 269, 137-144 (1984)

Schumacher, G. [SCH 1986] Harmonic maps of the moduli space of compact Riemann surfaces, Math. Ann 275, 466-466 (1986)

Schumacher, G. [SCH 1990] A remark on the automorphisms of the moduli space $\mathcal{M}_{p}$ of compact Riemann surfaces, preprint (1990)

Siu, Y.T. [SI 1980] The complex analyticity of harmonic maps and the strong rigidity of compact Kähler manifolds, Ann. Math. 112, 73-111 (1980)

Siu, Y.T. [SI 1986] Curvature of the Weil-Petersson metric in the moduli space of Kähler-Einstein space of negative first Chern class, Aspect of Math. 9, Vieweg Verlag, Braunschweig, Wiesbaden, 1986, pp 261-298

Siu, Y.T. [SI 1987] Lectures on Hermitian-Einstein metrics for stable bundles and Kähler-Einstein metrics, Birkhäuser-Verlag, Basel, Boston 1987

Teichmüller, O. Gesammelte Abhandlungen, Collected papers, (Ahlfors, L.V. and Gehring, F.W. edts.), Springer-Verlag, Berlin, Heidelberg, New York, 1982

Teichmüller, O. [TE 1939] Extremale quasikonforme Abbildungen und quadratische Differentiale, Preuß. Akad. math. Wiss., nat. Kl. 22, 1-197 (1939)

Teichmüller, O. [TE 1943] Bestimmung der extremalen quasikonformen Abbildungen bei geschlossenen Riemannschen Flächen, Preuß. Akad. math. Wiss., nat. Kl. 4, $1-42,(1943)$

Teichmüller, O. [TE 1944] Veränderliche Riemannsche Flächen, Deutsche Math., 7, 344-359 (1944)

Tian, G. [TI 1987] On Kähler-Einstein metrics on certain Kähler manifolds with $C_{1}(M)<0$, Invent. math. 89, 225-246 (1987)

Tian, G., Yau, S.T. [T-Y 1987] Kähler-Einstein metrics on complex surfaces with $C_{1}>0$, Comm. Math. Phys. 112, 175-203 (1987)

Todorov, A. [TO 1988a] The Weil-Petersson geometry of the moduli space of $S U(n \geq$ 3) (Calabi-Yau) manifolds I, (preprint)

Todorov, A. [TO 1988b] Weil-Petersson geometry of Teichmüller space of Calabi-Yau) manifolds II, (preprint)

Tromba, A.J. [TR 1986] On a natural affine connection on the space of almost complex structures and the curvature of the Teichmüller space with respect to its WeilPetersson metric, manuscripta math 56, 475-497 (1986)

Tromba, A.J. [TR 1987] On an energy function for the Weil-Petersson metric on Teichmüller space, man. math. 59, 249-260 (1987)

Varouchas, J. [VA 1984] Stabilité de la class des variétés kählériennes par certaines morphismes propres, Invent. math. 77, 117-127 (1984)

Varouchas, J. [VA 1989] Kähler spaces and proper open morphisms, Math. Ann. 283, 13-52 (1989) 
Weil. A. On the moduli of Riemann surfaces, Coll. Works [1958b] Final report on contract AF 18(603)-57, Coll. Works [1958c] Module des surfaces de Riemann, Séminaire Bourbaki, No. 168 (1958)

Wolf, M. [W 1989] The Teichmüller theory of harmonic maps, J. Diff. Geom. 29 449-479 (1989)

Wolpert, S. [WO 1983] On the homology of the moduli space of stable curves, Annals Math. 118, 491-523 (1983)

Wolpert, S. [WO 1985a] On the Weil-Petersson geometry of the moduli space of curves, Amer.J. Math. 107, 969-997 (1985)

Wolpert, S. [WO 1985b] On obtaining a positive line bundle from the Weil-Petersson class, Amer. J. Math. 107, 1485-1507 (1985)

Wolpert, S. [WO 1986] Chern forms and the Riemann Tensor for the moduli space of curves, Invent. math. 85 119-145 (1986)

This article was processed by the author using the $\mathrm{T}_{\mathrm{E}} \mathrm{X}$ macro package from SpringerVerlag. 